\title{
VJING: A NEW ZEALAND CASE STUDY
}

By

Naomi Lamb

A thesis

submitted to the Victoria University of Wellington in fulfilment of the requirements for the degree of Masters of Design

Victoria University of Wellington

2010 


\begin{abstract}
This thesis analyses some key factors of what is often considered a marginalised cultural and creative practice, VJing. The term VJ historically refers to Video Jockey, though in its own right $\mathrm{VJ}$ can be used to umbrella a number of various practices that utilise live visual media performances. This study is based on the researcher's active participation in the New Zealand (Kiwi) VJ scene for over thriteen years. Chapter One introduces some of the traditional principles and factors that are still in use in contemporary VJ practices, as well as the characteristics of the Kiwi VJ scene. Chapter Two outlines and analyses an experiment that takes these factors and characteristics into consideration. The case study utilises peer to peer information gathering through an experiment designed to generate a disruption to the intuitive mode of working to which four veteran VJs have been accustomed. The case study experiment sought to isolate key factors that impact on content creation and selection for live VJ mixing that utilise traditional principles. During the analysis, the following factors were identified: time, sight, memory, finance, environment, technology, availability, efficiency, compatibility, loops, silence, form, rhythm, movement, shape, colour and music. The experiment centred around a sample library of original content that the four VJs were asked to respond to, both through conversational dialogue and a live VJ performance. The DVD contains the results of the individual performances.
\end{abstract}




\title{
Acknowledgments
}

\section{Academic Support}

\author{
Morgan Barnard \\ Deborah Laurs \\ Thierry Jutel \\ Geoff Stahl
}

All the academic staff and fellow students at VUW school of design for their support

\author{
Pilot test and case study VJs \\ Grayson Cooke \\ Johann Nortje \\ Raylene Beals \\ Tim Budgen \\ Dave Isdale
}

The extended Kiwi VJ community.

\section{Music acknowledgements}

Michael Hobbs (Boofa), Dave Schofield, Pitch Black, Ben Horton, Blue Monk, Tom Cosm, Michael Hodgson

All my friends and family who have given moral support and encouragement. 
Abstract

Acknowledgements

Table of contents

\section{Introduction}

\section{Chapter 1 : Context}

- What is VJing?

- Predecessors

- Traditional VJing

- Contemporary VJing using Traditional Principles

- Club VJing

- Personal VJ or Concert VJ

- Audio Visual Artist

- Live Cinema

- Community

- Kiwi VJs: The development of VJing in New Zealand

- Kiwi VJs: Content and form

- Kiwi VJs: The community

\section{Chapter 2 : Case Study}

- Purpose and justification

- Sample library

- Music selection and justification

- VJ selection process and rules

- Experiment and analysis

- Sight memory and time

- Finance

- Technology

- Efficiency

- Environment (context)

- Silence, rhythm and motion

- Form: shape and colour

- Loops

- The DVD in context 
- Sample A

- Sample B

- Sample C

- Screen Grabs

- Conclusion

References

Appendix 1

Appendix 2 


\section{Introduction}

This thesis explores VJing as a process-based practice and provides an overview of the development both globally and locally. VJing is a relatively new creative practice that is often hard to define, as many practitioners participate within different contexts in which live video performance can be presented. While the initial stages of this thesis are broad in scope as it tries to locate a trajectory from a global context to a local one, it leads to the discussion of a specific case study conducted within this research. The overall objective of this thesis is to identify some key factors within the practice of live video art performance, in particular the practice of VJing.

I have always had a love of moving image and art - and a love of music. I entered into VJing in the late 1990s while completing a BFA, in what was called Computer Art at the time, at the Dunedin School of Fine Arts. Using a computer to make art created a displacement of my initial intuitive mode of working with my hands to produce work. Controlling and manipulating images with a mouse did not offer me the tactile experience I was used to. Thus, in order to retain an immediate and tactile relation with my work, I transferred my personal filmed footage and images created in 3-D Studio Max onto VHS tape and using an analogue editing suite I mixed these sources together, using four VHS players, an edit controller and a Panasonic MX 50. Improvising with this hands-on approach to technology and jamming material to music was a way to relax and to generate a new experience with the images I had created. I wanted to see changes happen in real time rather than wait for computers to do long renders in order to see the results of my labour. During this experimental time in the editing suite, I was unaware that many other practitioners within New Zealand and around the world who called themselves VJs had been doing the same. Since then, the practice of VJing has led me to explore various different and dynamic ways of recontextualising my video art while working in many varied situations, locations and contexts. Thus it has enabled my work to reach a variety of audiences that I would not have been able to reach had I confined my work to the art gallery context. My VJ practice has grown in New Zealand through performing at festivals, clubs and other music events and in particular within the outdoor dance party scene. It is these scenes upon which I concentrate my investigations.

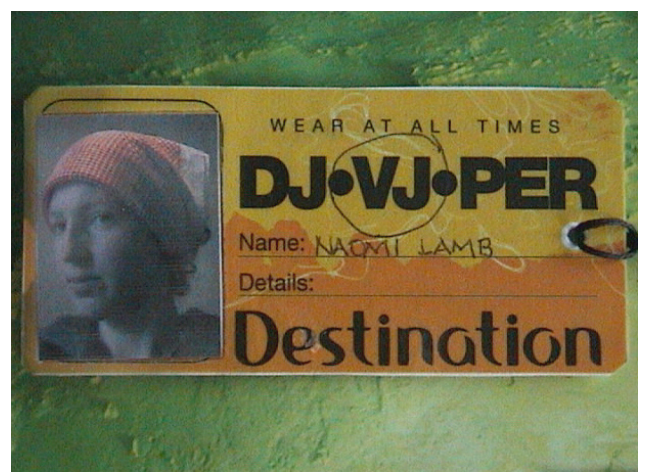

Figure 1: Festival pass 2004. Photo: Author

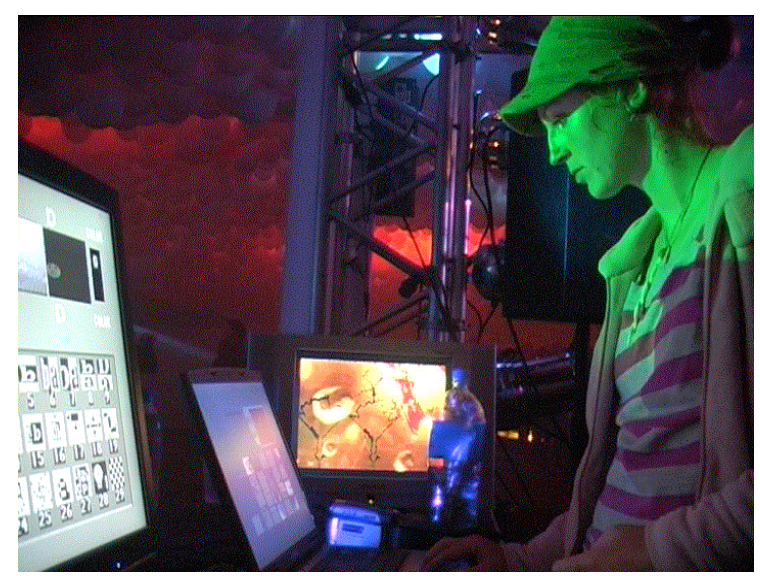

Figure 2: VJing at Cannan Downs Festival 2007. Photo: Author 
While continuing an art practice that ran in partnership with VJing, I was confronted with a surprising lack of knowledge in the general public and arts institutions of what VJing actually entailed. In addition, as information started to circulate about international festivals, books, and various art and performance practices that linked into the craft of VJing, I was often disappointed that many in New Zealand would reference these rather than any local works or practices. I realised that it was the lack of published material on the subject of VJing in New Zealand that keep it from being referenced and used locally. In my research I have only found one published article that attempts to speak directly about VJing in New Zealand. This publication comes from a personal perspective given by Michael Hodgson (Hodgson, 2006). Between 2000-2005 I cofounded and directed an initiative called Project Shown* (project shown*, n.d.), to support emerging video artists and VJs, and in 2007, I set up Kiwi VJs (Kiwi VJs, .n.d.) on Facebook as the first online community site for VJs from New Zealand to connect with each other.

My motivation for entering into a Masters by research programme was initially to explore different contexts in which VJing could be presented. Prior to designing this case study, I created three different performances that focused on bringing the craft of VJing to the foreground rather than as a visual backdrop as it is often used in performances. While these performances were successful in reaching new audiences, I kept coming back to the realisation that to offer a real contribution to academic study and to make a contribution to the New Zealand VJ scene, I needed to provide an overview of its cultural history. Moreover, I chose to conduct a study that uses a collaborative approach, something typical of the practice, in order to isolate the key characteristics of the New Zealand VJ scene. Perhaps due to the lack of any real significant critical discourse in published format, there are many uncomfortable associations with the word VJ, even among VJs. This craft is still often considered solely as a visual backdrop to late night raves. One of the key goals of this thesis was to formulate a case study which would demonstrate the required skills, craft and creative process of VJing. While there are different forms of VJing, this thesis focuses essentially on "traditional" VJing.

The first chapter provides a context to VJing by offering a sense of the historical fascination with harnessing and manipulating moving images. It also offers a global overview of the development of VJing by introducing information from the existing literature. The second half of Chapter One introduces for the first time an attempt at an overview of the VJ scene in New Zealand.

Chapter Two discusses the design, process and analysis of a case study to investigate the key factors that impact on content creation for VJ mixing in New Zealand. This research is significant because it focuses its scope within the New Zealand VJ community, which currently has little formally published investigation. It has been through my involvement in this community that I continue to learn and evolve my practice, moving through technology and software changes. Much 
of the community moves through these changes together offering information and resources to support each other. Therefore, this case study aimed at replicating the forms of collegial and creative collaboration that is characteristic of the Kiwi VJ scene. Four VJs, who are long serving practitioners in the community, and whose work has grown from the traditional VJ practice, were selected and agreed to participate to this investigation.

The motivation for the case study was to conduct an experiment that would identify the underlying factors impacting on content creation for live mixing or traditional VJing. The findings of this thesis on the process of content creation, selection and performance, are based on individual conversations and interviews. The experiment described in Chapter 2 demonstrates that my library of samples provided a catalyst for the four VJs' self-reflexive analysis of their practices. This thesis will demonstrate that this form of inquiry, based on making VJs react and respond to someone else's library of samples, was successful in generating useful information which could not have been gathered through interviews only.

The DVD contains the recorded outputs of each VJ's mix. These are arranged in a grid. This arrangement was constructed for the purpose of a public screening held before the analysis. The DVD screened at the end of my presentation exploring VJ contexts. This was held as part of a MIC Short Fuse programme in Auckland in 2009.

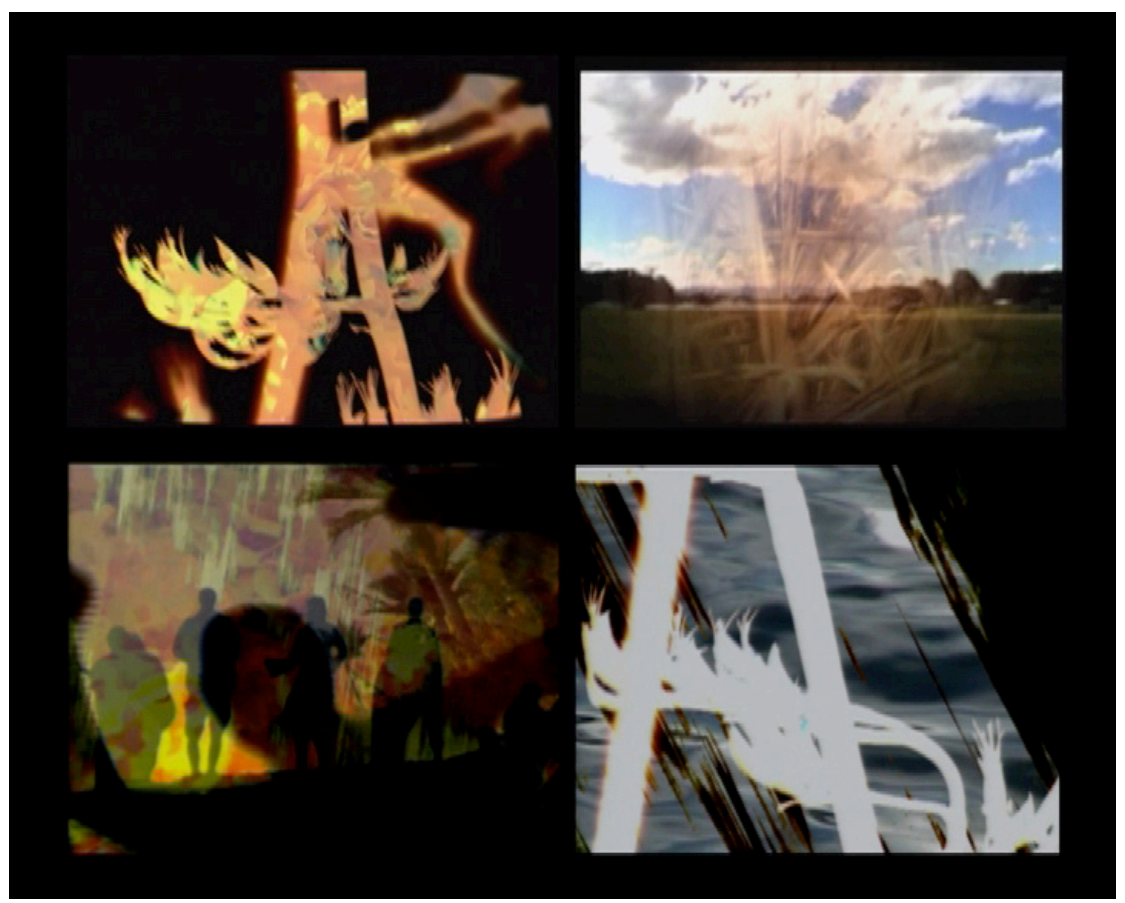

Figure 3: Screen grab of DVD. Photo Author 


\section{CHAPTER 1}

\section{What is VJing?}

Across cultures and through the ages there has been a long fascination with enhancing stories, events, environments and experiences with moving images. This might take the form of simple movements and shapes, or the creation of multi-sensory immersive environments. VJing takes this intent, magnifies it, and embraces the latest technology. It is the real-time mixing of images from carefully selected content for a public audience, most commonly in response to music or other performative arts. Paul Spinrad describes this well: "VJing is the type of performance that combines the visual possibilities of filmmaking with the improvisational pleasures of jazz" (Black \& Cook, 2003).

The purpose of this chapter is to show that VJing is a practice that is hard to categorise, due to the fact that it relies on technology and factors such as the 'scenes'. As with all contemporary media, VJing is constantly evolving, both in terms of its history and day-to-day logistics, with interwoven elements of location, equipment and aesthetics. This chapter looks at some of the antecedents to VJing which highlight the fascination with capturing and presenting live motion, while providing some of the key characteristics of the form. Then it looks at the development of the principles of traditional VJing followed by a discussion of some complementary practices in the contemporary VJ scene. The chapter begins with the general, globally accepted view of the VJ scene and then looks at the New Zealand scene in particular.

\section{Predecessors}

VJing as a concept is nothing new. There are a number of precedents, ranging from the early $1600 \mathrm{~s}$ to the late 1960s, that combines story-telling, moving images, and different forms of technology.

One form of enhancing a story using projections is the traditional Indonesian performative art form, Wayan Kulit [sic] (Mikela, 2006). It uses flat layered, often ornate, cut outs of figures with moving parts. These are manipulated by the puppeteer using long sticks set in front of an oil lamp allowing the puppets to cast black silhouette shapes onto a white screen.

In Europe in the late 1600s, a device called the 'Magic Lantern' was used to entertain audiences (Mikela, 2006). It had glass plates that could be directly painted on and moved in front of an oil lamp that rear-projected these "slides" onto a screen for spectacle exhibitions (Faulkner, date, p. 16).

In 1881, after the discovery of visual latency "in which the image remains for a very brief period of time on the retina of the eye" (Faulkner, 2006, p. 16), Jozef Plateau designed a single viewer device called the phenakistiscope. This was a circular disk with a series of images painted on it at 
different stages of motion and when turned it created the illusion of a moving image (Faulkner, 2006; Flipbook, n.d.).

Pioneering photographers Etienne-Jules Marey and Eadweard Muybridge were instrumental in the capturing of motion (Horrocks, 2009, p. 16). Marey invented the 'photo gun' in 1882: "This camera was constructed in the form of a rifle that was able to take twelve photographs per second" allowing Marey to follow a moving subject (Horrocks, 2009, p. 16). On the other hand, Muybridge used as many as twenty-four single cameras that were set off sequentially in his attempts to perfect capturing motion (Rush, 2005, p. 15; Horrocks, 2009, p. 18).

The development of flipbooks turned these scientific photographic studies into entertainment. Samples of these early photographic sequences were layered over the top of each other and, when the pages were flipped quickly, like the phenakistiscope, it gave the illusion of motion. The flipbook could be physically flipped though at different speeds and either forward or backwards. (Flipbook, n.d.). While photographic inventions revealed motion in a static way, flip books brought them to life.

When cinema emerged in the mid-1890s this new development meant that further experiments in filming and presenting moving images could be explored (Faulkner, 2006, p. 17), both in how the cameras were operated and the images edited. The cameras were operated using hand cranks, and the speed at which the crank was tuned dictated the speed of the playback motion, often with comical results (Horrocks, 2009, p. 21). Other experiments and techniques led to exploring montage. The 1929 film by Dziga Vertov, Man With a Movie Camera, shows the accumulation of these techniques, making use of fast repetitive cuts, exaggerated motion, time based and spatial montage (Manovich, 2001), juxtaposed subject matter, different camera angles and focal points and shots such as close-ups to add drama. Rhythmic elements within the frame such as double exposures or overlays, slow motion, reversed motion, and stills were also used in conjunction with these techniques to elaborate this montage process.

The 1950s brought in the development of overhead projectors [OHP] and in turn these were used in light shows, which were also known as Wet Shows or Liquid Shows because the artist would pour transparent liquids or oils onto the OHP lens to cast a fluid moving light (Spinrad, 2005, pp. 1920). These shows continued into the 1960s in combination with experimenting with film, mirror balls, liquids, slides and UV lights (Spinrad, 2005, p. 20). It was during this time 'scenes' developed that enabled further radical visual experiments in manipulating light and image in live, often improvised, ways in response to the other goings on, like dance, music or so-called "happenings". These light shows extended the psychedelic immersive experience that sprang up in association with the use of hallucinatory drugs such as LSD (Spinrad, 2005, p. 20; Faulkner, 2006, p. 15). They offered a multi-sensory overload experience by using much of the technology of many of the previously mentioned visual developments for public projection. 
Collectively these early developments highlight some key factors that are still in consideration when making a VJ mix. These include layers (shadow theatre), montage (silent film), motion (photography, flipbooks, cinema), liveness (flipbooks, shadow theatre), loops (flipbooks), improvisation (wet shows, flipbooks), content for the screen (film), projections, (magic lantern, light shows) remixing (flipbooks), and rhythm (silent film).

Although Spinrad (2005) and Mikela (2006) claim that contemporary VJing grew out of these forms and techniques, it was the invention of the VHS camera and VHS player that really opened up the field for VJing. Since VHS has now been superseded, it is possible to categorise VJing into traditional and contemporary forms.

\section{Traditional VJing}

In the late 1970s, VHS technology started to become affordable to non-professional users, thus making it into the hands of many video artists (Spinrad, 2005, p. 21). Some video artists had started using this technology earlier, for example Nam June Paik in the 1960s with the Sony Portapack, an early video camera (Spinrad, 2005, p. 21). However, this equipment was still fairly expensive by today's standards. Amateur artists could afford a few pieces of equipment, but, in many cases, only professional dance clubs could purchase and make use of multiple televisions or screens and VHS players. Places like the Peppermint Club in New York that featured electronic musicians and DJs offered experimental video artists a dynamic place to showcase their work (Spinrad, 2005, p. 21). As well as the electronic music scene sharing a like-minded passion for technology it was also that the electronic musician or DJ was no longer very interesting to watch (compared to a full live band). So out of necessity the VJ or video artist provided visual backdrops to enhance the environment and keep the crowds visually stimulated. As opposed to cinema or television, the audience no longer had their full attention focused on the screen, which meant it was no longer necessary to have one particular focal point. Because audiences were dancing, they were also unlikely to watch a performance from beginning to end, thus enabling the VJ to further experiment with fast paced mixing and juxtaposing of various different types of content. It was with the developing electronic music scene and these clubs (Hodgson, 2006, p. 148) that the emerging VJs had a real place to develop their skills.

The 1980s brought in another piece of equipment that would have paramount influence on the development of the traditional VJ practice: the video mixer. Mixers such as the Hardware Fairlight Mixer or the Panasonic WJ-MX50 enabled the VJ to add real-time effects to content and allowed for content from multiple VHS decks to be fed into the mix simultaneously. It was this combination of using mixers and multiple VHS decks that established the traditional techniques such as multiple layering, 'keying' or masks, spatial montage, sampling and looping media many of which are still in use today. Using a mixer the VJ could have as many as four VHS sources to mix with at any one time. The resulting output was achieved using a straight cut or cross fade. 
Two layers could be simultaneously played together, again with the cross fade or using a luma key or a chroma key, where either the black or white (luma) or dominant colours (chroma) of an image could be 'keyed' out, like a silhouetted figure, and replaced by the other source's image. The builtin effects of these mixers allowed much more real-time control of content manipulation. These included the paint effect, mosaic, picture in picture, mirroring, grids, (multiple screens within the screen) or shapes that affected the wipe or cross fade (User Manual, n.d). Different configurations could include the use of a live video signal to provide haphazard effects such as video feedback where the image was fed back into itself and 'bleeds' and dies, or creates fractal like patterns of motion and colour. All these transitions and effects were physically controlled by the use of buttons, knobs and sliders so the VJ could improvise with layering, cutting, mixing and tweaking the content rhythmically in response to the music.

The VHS tapes had durations of 10 minutes to 3 hours. A mix tape could contain sample content that was taken off television, specifically filmed, or appropriated from feature films. Simple looping graphics could now be created thanks to introduction of "The Graphical User Interface [GUI] by Apple Macintosh in 1984" (Faulkner, 2006, p. 19). Mix tapes could be prepared in advance using equipment such as these early computers, mixers or editing controllers that were starting to come out of outdated television suites. A VJ would have vast collections or libraries of these mix tapes and the combination of these with multiple VHS players and a mixer meant that a diverse range of visual content that could be mixed and affected from once single control station, albeit a large and expensive one.

\section{Contemporary VJing}

In the 1990s, during the shift from VHS to today's laptop, the DVD emerged. Player and authoring software enabled VJs to better access their sampled content through the use of chapters and repeat functions. This, combined with the introduction of new, faster and more affordable computers, with software for both creating and editing content, cut down on the labour required to create loops on a VHS tape and provided the ability to select content faster and easier on the fly. However, with the new dedicated VJ software (made by VJs for VJs) all content could be now accessed through the computer hard drive.

During the late 1990s and early 2000s software such as Resolume, Arkaos, and Modul8 emerged to provide the $\mathrm{VJ}$ with all the ability of mixing content using the traditional principals, of layering, looping, montage, keying, spatial montage and effects. The inclusion of MIDI and BPM syncing made it easier for the $\mathrm{VJ}$ to have the content respond rhythmically to the music and the traditional knobs, buttons and sliders were replaced by a mouse. The mixers became smaller as well, with companies such as Endarol producing mixers like the V4 ( figure 4) which incorporated all the mixing possibilities of older mixers but at half the size. 


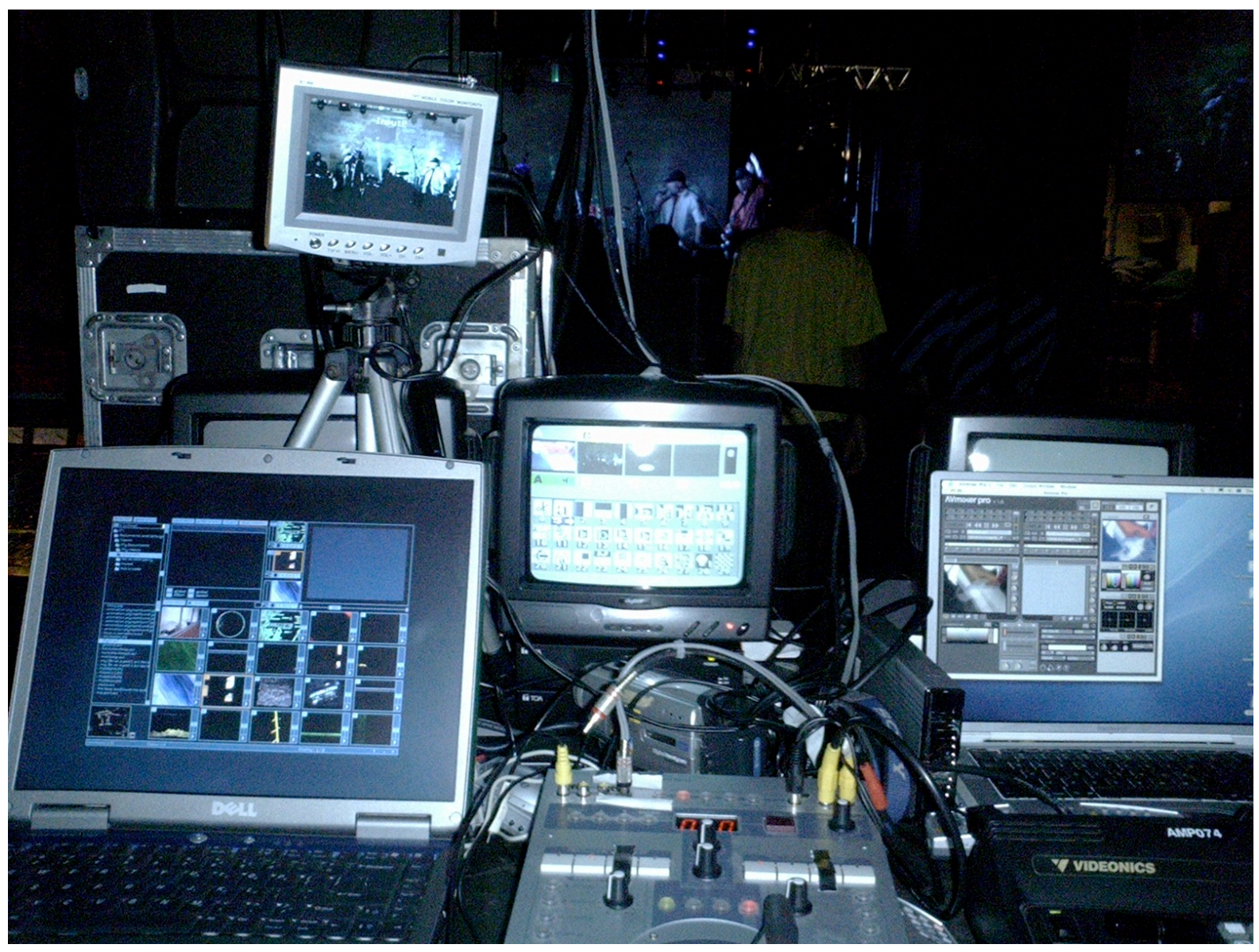

Figure 4: VJ rig 2005, mixer in the centre is V4. Photo: Author

These developments enable the contemporary VJ to access large libraries of content that are specifically pre-prepared into "samples" or more commonly known as "loops" from within the VJ software so that all a VJ needs to start out is one laptop with a direct link to a projector. The ongoing developments of computers, software, and other instruments like MIDI keyboards and mixers mean that the configuration of a VJ rig is never uniform. There are a variety of ways and places a VJ can perform. Even today it is rare that a venue would have any standardised equipment for presenting visuals so VJs need to either own or hire the equipment needed for each performance. This means that many VJs have a good understanding of not only collecting and compiling content libraries but also many of the various forms of equipment and methods used for content delivery.

The following examples of contemporary VJ practices exhibit similarities with traditional VJ in that they may use libraries of content (loops), layers, montage, motion, liveness, improvisation, content for the screen, projections, remixing, and rhythm. There are many more examples of where VJing can be used to present live visuals than offered here and the titles given to each genre are only broad umbrella terms. 
Figure 5: Example of Club VJing. Photo: Author

\section{Club VJing}

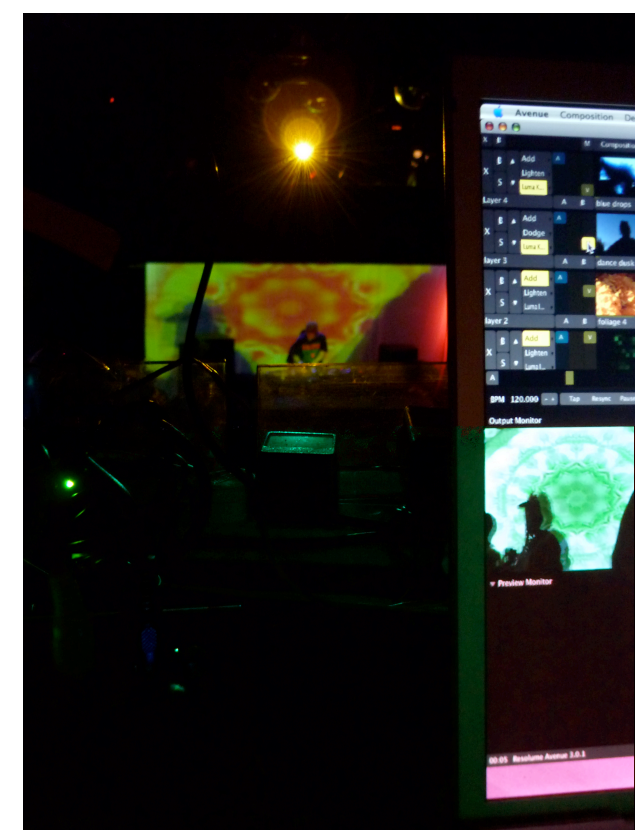

Club VJing as a genre has had a consistent progression in the history of VJing since it was these club environments that helped foster the development of this practice. The role of the club VJ is to offer a constantly evolving visual stimulus to entertain crowds and support the musical selection of the DJ. These visuals are often projected behind the DJ or in and around other areas of the club, such as video walls or side panels, to provide an immersive environment for the event. Often the club VJ works alone and can be the sole mixer from $10 \mathrm{pm}$ and until the time when the club closes, usually round $6 \mathrm{am}$. It is gruelling work but exhilarating at the same time. The content a club VJ uses varies and reflects the aesthetic preferences of the $\mathrm{VJ}$, the genre of the music, the theme of the club or the needs of the DJ or promoter. Therefore the VJ needs a large library of loops to mix from and a VJ that is in regular employment will need to add to this library continually just as a DJ needs to continually update his or her music selection. This type of VJing is often the most improvisational as VJs might not be aware what music selection the DJ will play. The VJ's library of loops needs to be extensive and varied so that there is some correlation to the music and that the mix can be sustained over a very long period.

\section{Personal VJ or Concert VJ}

A sub category of the club VJ is the personal VJ (Black \& Cook, 2003) or concert VJ (Virkhaus, 2006, p. 37), where the VJ provides backing visuals for a band or similar type concert. This can be similar to club VJing but more often the visuals are selected in response to each individual song, where an opening or closing and selected images may illustrate the lyrics or band's identity. The $\mathrm{VJ}$ is autonomous but his or her role is like any other member of the band as the visuals created are rehearsed and made in combination with what the band's overall vision is. The performance is still live and dynamic but often it is just repeated the same each time. Often at the higher level of 
production these VJs update their skills and visual content regularly so they are able to deliver the best possible visuals for contemporary audiences.

\section{Audiovisual Artists}

Unlike the club VJ or concert VJ who is separate in his or her role from the band or DJ, the audiovisual artist combines both sound and vision. The artist composes the visual to support or supplement the music in a technically incorporated way. This can be simply combining both audio software with real-time video software or can be much more complex involving multiple devices. While the performance is live, the audio and visuals are often designed or rehearsed together. The main focus or skill set may be from an audio perspective and the video is incorporated and sampled in the same way or the video could be abstract and adding a visual score with depth and simplicity that supports the music, adding to the sensory experience.

\section{Live Cinema}

Live cinema (Mikala, 2006) takes a departure from the above genres due to the different context in which these types of performances are often developed. While there are many similarities to the types of VJing already mentioned such as using similar hardware and software to accesses a library of loops to create a montage of layers in real-time, it differs from club VJing in that it often has a defined timeline and the audience is usually stationary. Generally, this is a slower-paced experience, sometimes incorporating experimental or non-linear narrative. Live Cinema presents itself to audiences different from that of the club VJ, often in art galleries or in theatres, and the relationship to the audio, soundscape or music can be considered a partnership rather than one leading the other. While Live Cinema is still a real-time performance and can be improvised, it is often rehearsed or had pre-production consideration.
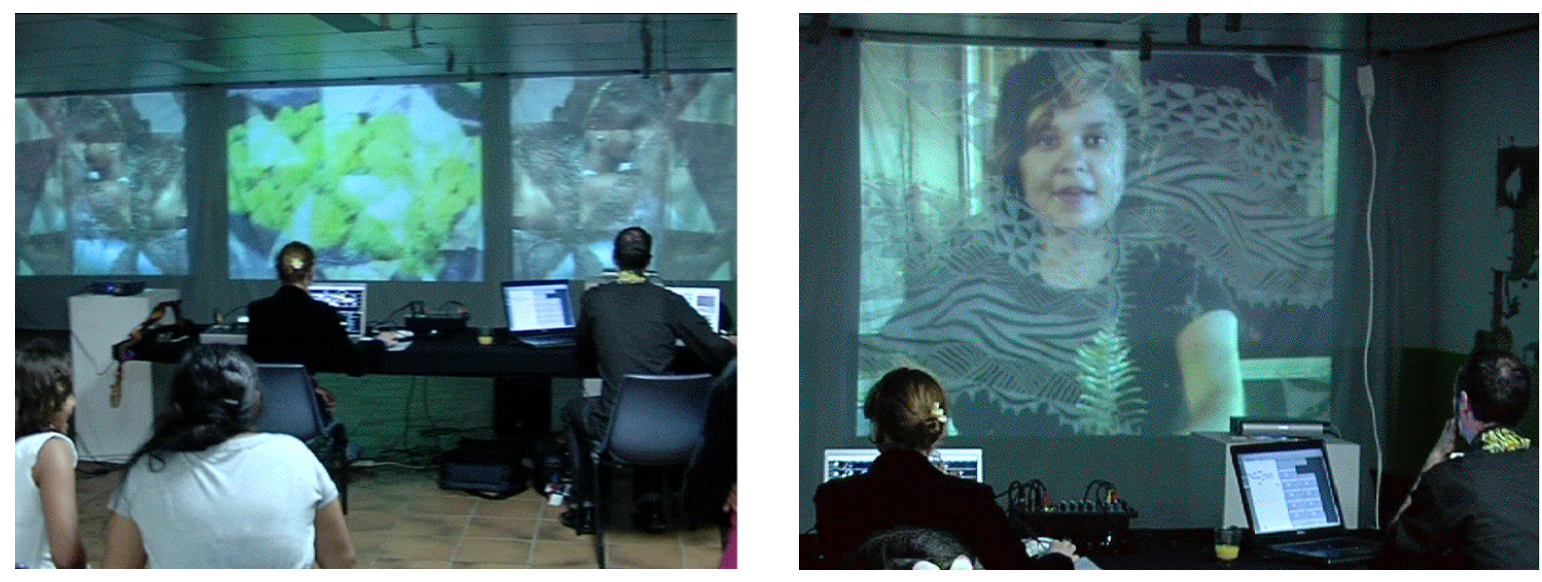

Figure 6 a, \& b: Installation view of live cinema performance Pacific Storms, Bundaburg 2009. Photo: Author 
This has not been an exhaustive list of where the skills of a VJ can be practised or observed, but the forms discussed above constitute some of the most recognisable practices with in the VJ community.

\section{Community}

VJing has a short but dynamic history and the number of people becoming interested in the practice of VJing is increasing (Hodgson, p. 149). In its early days connecting with other VJs was very localised. Music festivals and other multi-night events that needed numerous VJs provided one of the few ways for practitioners to network and share ideas and skills. It was often a very isolated existence especially if the VJ resided in a town or country that did not have many people exploring this medium. In addition there was very little documentation of VJing and VJ culture. However, since the emergence of Web 2.0 there are now many opportunities to connect with and become part of the VJ community both locally and globally (Faulkner, 2006, p. 19). VJs are naturally very social, as working in environments such as festivals and clubs does not generally suit introverts. VJs have adapted well to networking, sharing skills and communicating in the online environment due to their social nature and having a like-minded passion for technology and visual media. The following examples are just some of the many avenues that can be followed to extend both knowledge and participation into VJing and VJ culture.

There are online forums that enable VJs to share information and ask the community for advice such as VJ central which was one of the first online VJ forms and today has many active users. Companies that develop VJ software such as Resolume and Modul8 offer supportive online forums into their websites thus further building a community around their products.

Even though many VJs have their own personal websites, online communities such as Facebook attract VJs to connect either through direct connection through "friends" or though some of the many groups that have been created to support VJs such as Kiwi VJs, an open group for VJs from New Zealand to share information, or $V J$ node, a global Facebook group for further sharing of information.

While there may be other interest in VJing from other creative sectors, the VJ community is mostly steered by the most active members who work hard at helping educate, illuminate and enhance people's understanding of what VJing is and what is involved. The current published material on VJing includes works such as The VJ Book (Spinrad, 2005), VJing Audio-Visual Art + VJ Culture

(Faulkner, 2006), Live Cinema Unravelled (Jaeger 2006), and Live Cinema (Mikala, 2006), and an online journal, $V J$ theory (VJ theory, n.d), which are all produced and instigated by active members of the VJ community. Overall, then, while the global VJ community itself is highly active, the ephemeral nature of this performance art means there is very little representation in print media. 


\section{Kiwi VJs: The Development of VJing In New Zealand}

If there is little written about VJing internationally, there is even less on New Zealand VJing, with the Kiwi VJs Facebook page (Kiwi VJs, n.d.) one of the few online forums specifically targeted for New Zealand VJs. However, this does not mean that New Zealand does not also have a highly active and innovative VJ scene. New Zealand has its own early examples of artists who were interested in experimentation and performing with the moving image and who constitute some of the precursors to the contemporary VJ scene. Experimental film and kinetic artist, Len Lye, worked directly onto celluloid film from 1929 to create rhythmic images or visual music (Horrocks, 2009). In 1949, Cameraman Brian Brake teamed up poet/script-writer James K. Baxter, composer and pioneering electronic sonic artist Douglas Lilburn and painter John Drawbridge to create the film Aspiring (Scott, 2006). This film combined cinematic explorations of the landscape using montage, music and poetry. In the early 1970s intermedia artist Phil Dadson (Sonics from Scratch) started incorporating experimental video projections into stage and experimental improvised music shows. Collectively these few examples exhibit the use of moving image to enhance performance, to explore stories or to explore the medium in relation to itself and other art forms to construct new forms of expression.

VJing in New Zealand started to emerge in a similar way that VJing developed overseas. First various music scenes developed and required visuals for live shows. Interest in using live projections grew: "Many of New Zealand bands started to project $16 \mathrm{~mm}$ films over themselves during their gigs [and] we had our fair share [of events that included light shows that contained] oil wheels and trippy slides" (Hodgson, 2006, p. 148). These scenes and an interest in visuals were already in place when VHS came to our shores: "The VHS had a huge influence on what was to become 'VJ culture' in New Zealand" (Hodgson, 2006, p. 148). Thus traditional VJing in New Zealand was much the same as described overseas as it relied on a matrix of VHS tapes and video mixers. However, due to both New Zealand's isolated location and small population, often local VJs would either get new technology after that of America or Britain or this equipment would be much more expensive. So while VHS technology was starting to be explored by American VJs in the mid 1970s, here in New Zealand it was not until the mid to late 1980s that VJs started to explore VHS (Hodgson, 2006). With the combination of the growing music industry and the introduction of new technologies, the 1990s rapidly became a landmark in the landscape of New Zealand's live and electronic music scene with more venues and club nights to house touring bands, and with dance parties and raves becoming a regular feature in both urban and remote New Zealand. Jon Dix gives an overview of the live band scene from 1950 to 2000s in his book called Stranded in Paradise (2005). He explains that the 90s were a turning point in music heralding in the growth of the scene here (Dix, 2005, p. 283). Mike Hodgson also identifies the 90s as a strong point in New Zealand's VJ history with VJs becoming booked and billed alongside other musical acts (Hodgson, 2006, p. 148). 
The club scene in New Zealand has provided a significant and supportive context for the development of VJs. There are only token references to VJing within the electronic scene, but the movement has begun to be archived on social networks such as the Facebook group Christchurch 90s Rave Scene. The evidence collected on that group demonstrates that VJs were listed on advertising flyers and tickets from the mid to late 90s. Just like the clubs of New York that supported the growth of VJing, New Zealand clubs provided similar supportive environments to support and develop VJing. In addition to these clubs and select warehouse parties, and due to the proximity of the outdoors, the electronic dance scene started to expand into outdoor environments to hold events into New Zealand's expansive landscapes (Outdoor Styles, n.d). New Zealanders have a strong relationship with the outdoors with camping as a traditional holiday past time. Thus while the club scene was developing in the cities, a strong outdoor party scene was emerging in the outdoors, often involving multiple club promoters, managers, musicians, artists, DJs and VJs working together. VJing in the outdoors followed the same rules and employed the same technologies as those in clubs. Sound systems and diesel power generators could be taken to remote locations and the volume of the music could be louder than in the clubs, with no problems from noise control or neighbours. These events, variously called raves, outdoor dance parties or music festivals, could go on for many days and offered the perfect on-location lab environments for Kiwi VJs in which to work, expand their skills and network. As well as giving the DJs massive sound systems to work with, these locations provided unique opportunities for VJ designs. Screens would often have to be custom-built and could be any size or shape that resources would allow. The only restriction was the capacity of the projector. Screens could be built into or around the stages or in and on large marquees, and sometimes even the environment itself, cliff surfaces, grassy hills and rivers, would be projected on (Kiwi VJs, n.d.). These festivals, which can range in size from 500 participants to over 16, 000 (The Gathering, n.d.) offer some of the most extreme working conditions that anyone could experience, such as high mountain passes, limited access requiring transportation by foot, extreme and highly changeable temperatures, persistent rain or dry dusty conditions, no electricity, telecommunications or plumbing. 

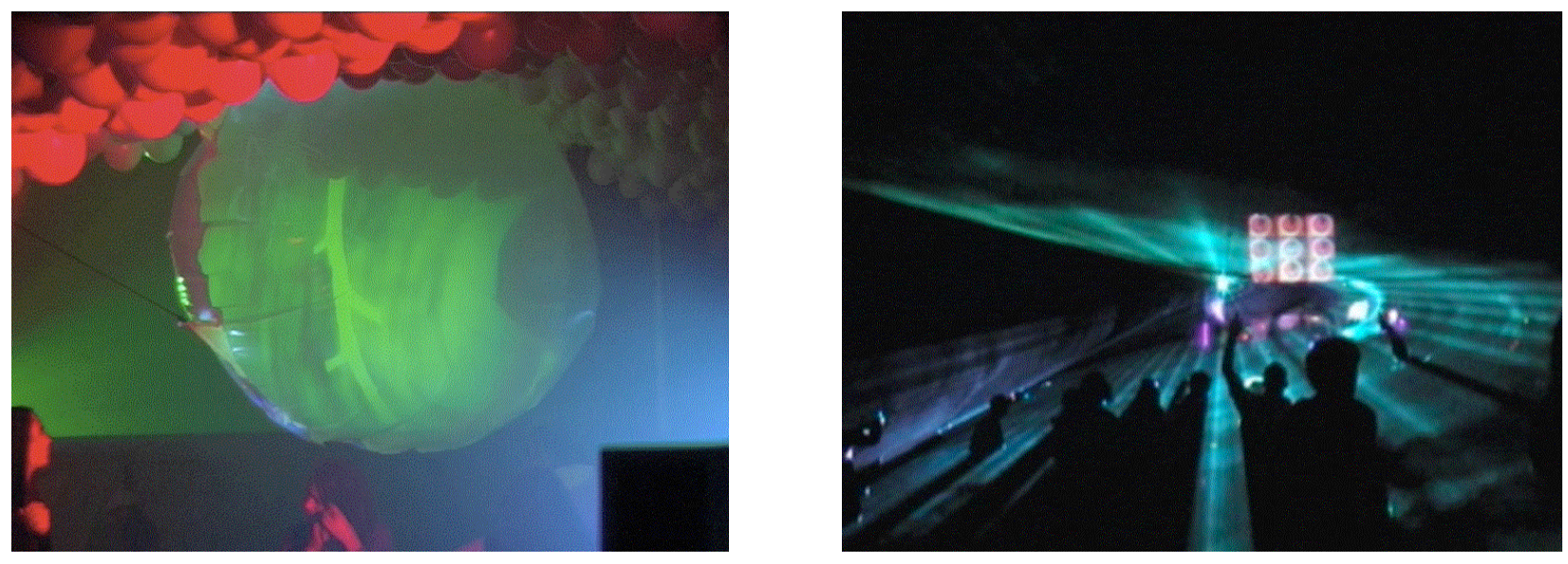

Figure 7: Installation view of projection surface at Cannan Downs Festival 2007. Photo: Author.

Figure 8: The Gathering countdown 2000-1. Photo: Author

Most artists drawn to these events saw their construction as multi-sensory installations. As well as often extreme conditions, these outdoor events provided a good place to meet with other likeminded practitioners from all over the country, and often these multi-day events were where a lot of VJs served their apprenticeships. Just like the clubs, it was these events that would be able to support VJs by providing the equipment necessary for large-scale shows. In fact these events were likely to provide more equipment than any club event. This meant that VJs also often had to work on rigs that were not familiar to them, but this again provided a learning opportunity. Thus VJs needed to contend with waterproofing huts and stages, wind ripping screens, and many days of camping, with little luxuries. They also needed to rig a lot of different video equipment into many different configurations to make the most of what was available in terms of resources and environment.

The event Entrain in 1995 (Christchurch 90s Rave Scene, n.d; Hodgson, 2006, p. 148; The Gathering, n.d.) was one of the first to use video projection in a large annual outdoor event. Later much of the crew from Entrain joined forces with other dance party crews to start The Gathering. The Gathering, which ran from 1997-2001, was one of the first major outdoor dance parties that could support up to thirty VJs on site to cover anywhere from two to six zones that needed visuals at this three night event (Hodgson, 2006; The Gathering, n.d). It was events such as The Gathering, and later Destination (Dance On A Rock, n.d), Alpine Unity (Alpine Unity, 2009) Splore (Splore, 2010) and Vision (Vision, 2001), which provided the space, time and resources that enabled VJs to explore and develop their individual aesthetics. 


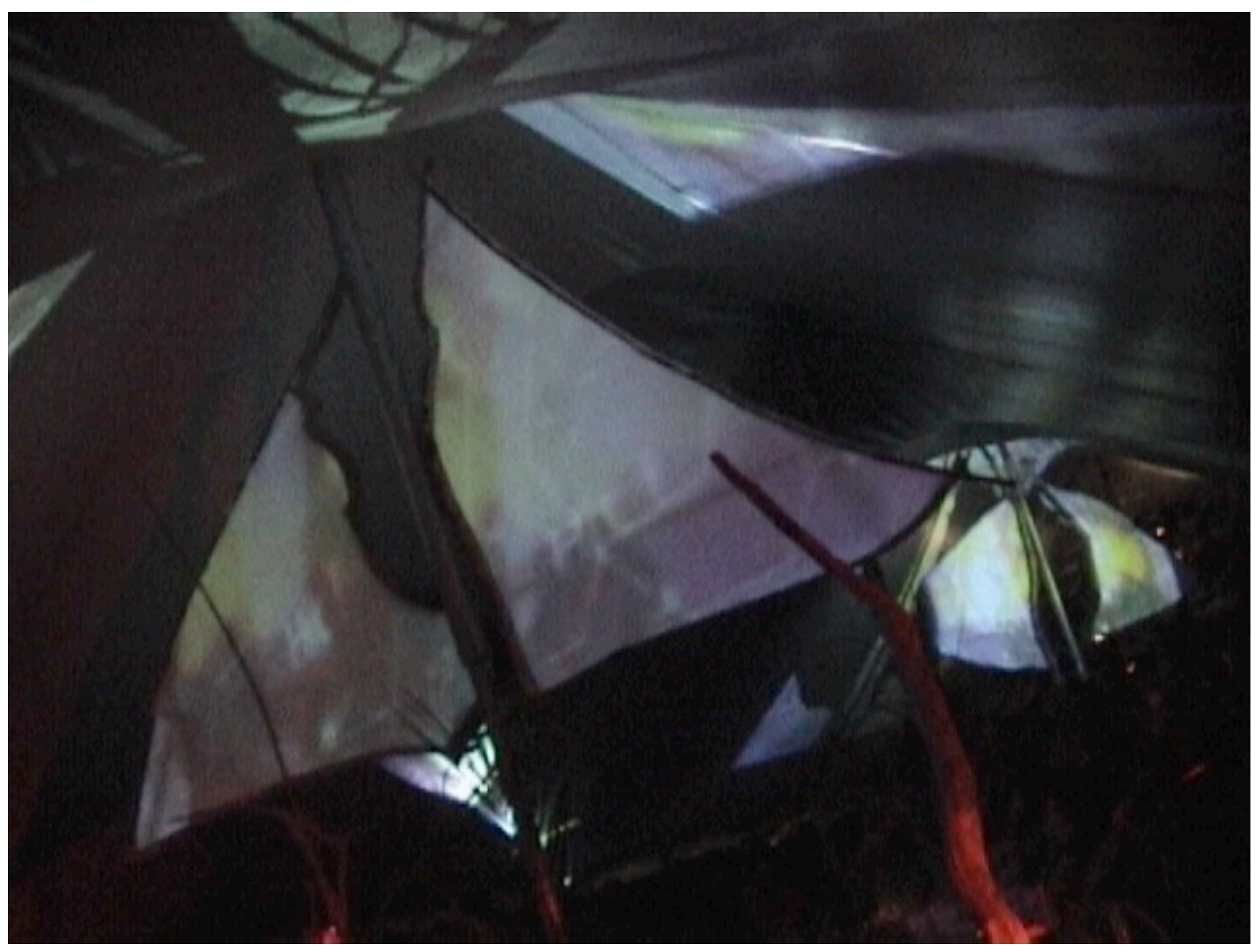

Figure 9: Installation view of dance tent at the Vision festival. 2003. Photo: Author

One of the key characteristics of the New Zealand scene was having the audience in the same location for a long period of time. The visuals need to change as much as the music, so a long festival requires lots of VJs, and VJs need lots of different content. At these festivals, the main stages often offered bands, and then there were other areas or large marquee tents, most commonly referred to as "zones", that contained similar types of music, such as house, hard core, drum and base, trance or ambient electronic or even curated sculpture trails and multi media installation sites. VJs could request slots, or be scheduled alongside a band or DJ, based on the complementary style of his or her content.

\section{Kiwi VJs: Content and Form}

VJs were wary of using copyrighted material. However, it was not unheard of that VJs would hire or copy movies from video stores. Incorporating imagery from popular culture like TV icons and animations and big budget movies was more likely to be seen in urban underground events, due to the ephemeral and non-commercial nature of these performances. However, in the outdoor dance party scene, the public attending these events tended to regard this as an opportunity to enjoy some escapist entertainment. Thus images of daily living, politics, work, pornography (because some of 
the events attracted families) and industrial imagery were almost always excluded. Rather, content such a abstract two-dimensional (2-d) and three-dimensional (3-d) animation and graphics, nature, landscapes, fire, water, macro and micro textures, and the use of a live camera were more prominent. A top VJ would collect samples of this original content through making it him or herself, most often through filming and editing or using computer graphics. This approach also meant that each VJ would develop his or her own aesthetic, through the choice of content and how he or she decided to mix it together using the available technology.

With computers becoming more popular for image creation and eventually for playback at the turn of the century, rigs often became a hybrid of VHS, DVD, different mixers, and an input for the VJs personal computer, using DVD or VHS as back up. The creation of content was influenced by each new piece of technology which became available. As mentioned above, a hardware mixer could be used to layer and add effects. That layering was further explored with the mixing of shortened loops from DVD chapters and, eventually, looping content played back through VJ software. One might think that after the introduction of the Internet and DVDs there would be more sampling of reproduced mainstream media. However, New Zealand VJs still did not have the ability to copy DVDs or download material as readily as one might from overseas, due to limited software compatibility and slow bandwidth. Even when this could be done, the quality of the image was usually poor. So for legal, practical, and aesthetic factors, most veteran VJs concentrated on making their own content.

\section{Kiwi VJs: The Community}

The VJ community relied on information sharing through a peer-to-peer network. It was during the outdoor parties that many of the conversations took place. Content creation was a common conversation within the $\mathrm{VJ}$ community at the time, with most VJs making sure that at least $80 \%$ of their library was original. However, as the interest in VJing grew with more parties and clubs developing in local areas, the need for VJing also developed and in a short time many VJs realised that it was just too time consuming to constantly update their content. Around 2001, discussions focused on seeking out and sampling content from other sources became a common feature at festivals. However by 2002, the growing number of outdoor events competing for the same number of ticket sales meant they became financially unsustainable. A subsequent decrease in outdoor parties meant fewer opportunities for VJing during the festival season. In addition, the cost of equipment was still relatively high and, for club and touring events, the visuals were usually the first to be cut from the budget. The demand for VJs dropped.

In an attempt to keep the $\mathrm{VJ}$ network alive and provide alternative places for proximal networking and using their skills, supportive VJ initiatives were set up during this time. Project Shown* (Project Shown*, n.d.) was one such initiative. This ran from 2000 until 2005 and provided a 
network for both VJs and experimental video artists to show their work in theatre screenings and art galleries. While based in the South Island, its network was far reaching. In August 2003 it hosted New Zealand's first three-day VJ symposium, attracting VJs from around the country to meet and share their skills with the wider audience that extended beyond the dance culture (Project Shown*, n.d). Also, at the turn of the century, Intersect (Intersect, 2009) was initiated in the North Island, which provided a regular "Plug and Play" type environment that facilitated group and individual VJ-related events, again with the intention of supporting the VJ community while attracting new audiences. From about 2006 there was a resurgence in the numbers of VJs coming into the scene or returning to it again (Hodgson, 2006). A number of new VJs came from the club scene in the cities, often attached to the genre of music they liked, or approaching VJing as an extension of their interests in new technology developments such as coding, or physical computing. With equipment also getting cheaper, this has enabled more events to revive the need for VJs.

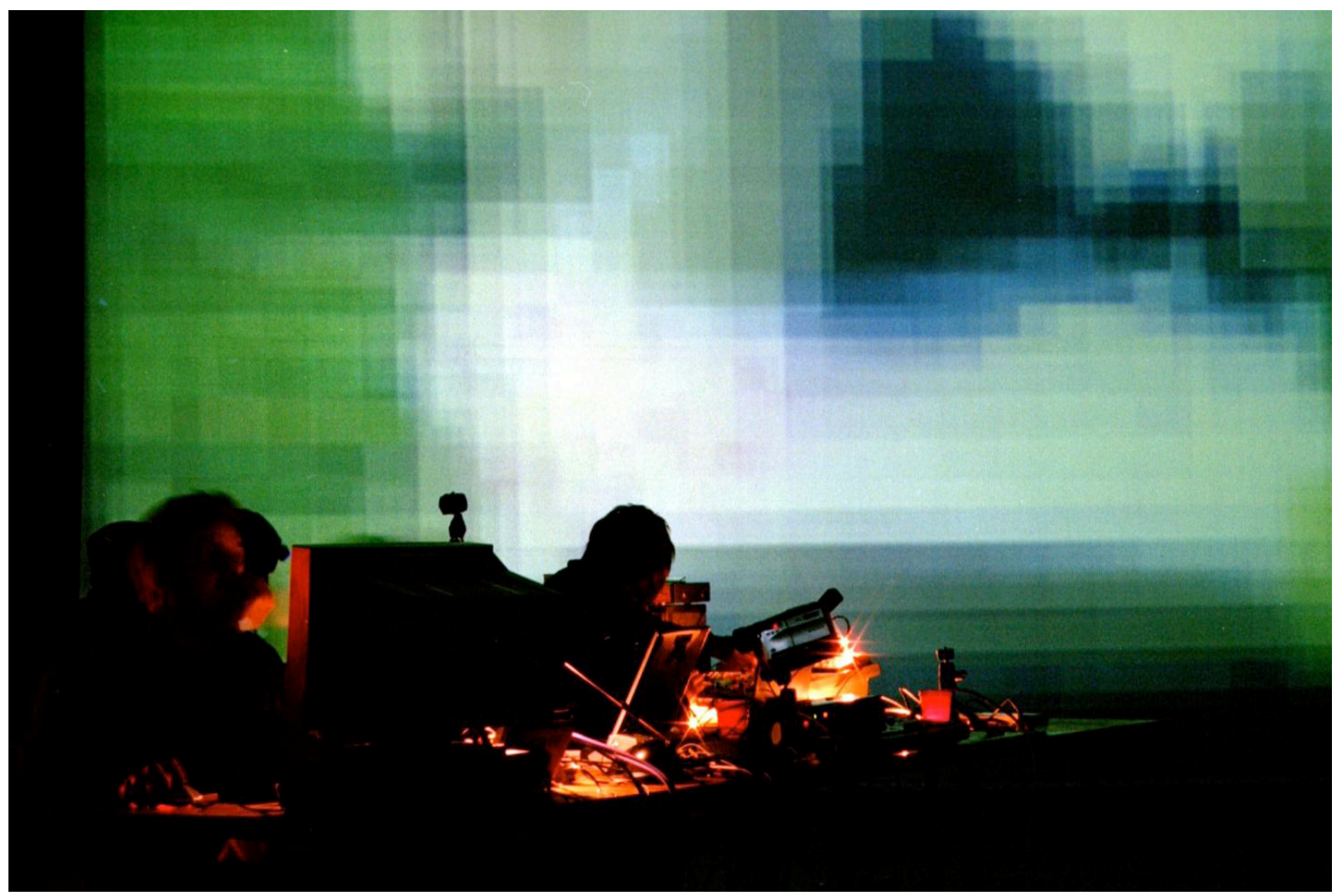

Figure 10: Project shown*'s New Environs VJ Symposium 2003. Photo: Robbie Forester.

\section{Kiwi VJs: The Contemporary Situation}

Kiwi VJs still have a strong presence in the electronic music scene with VJs touring with bands, DJs, or produced shows. The skills of a VJ can be diversified to accompany a wide range of live performances and Kiwi VJs have found themselves performing in art galleries, at corporate events 
and launches, for orchestras, in fine arts symposia, teaching in universities, developing software and VJ interfaces, as well as in continued clubbing and outdoor festival environments. The interest in VJing is growing as equipment becomes cheaper and the general public are becoming more familiar with what VJing is about. An additional factor that has influenced the resurgence of VJs is the global movement of remixing. While media copyright is still a contested topic, there are now more legal ways to appropriate visual content than ever before, most of which is available online. The bandwidth in New Zealand is faster now and better quality material can be downloaded and samples and made into loops. With the introduction of Creative Commons licensing, where the author of content can choose his or her own licence, VJs can access material that has a share and share like licence for the purposes of remixing (Creative Commons, n.d). Still, with the availability of new media available for VJs to sample, this content often needs additional treatment before it goes into the library.

VJing has grown from a range of visual performance precedents, with content strongly influenced by the technology and the 'scenes' in which the VJ performs.

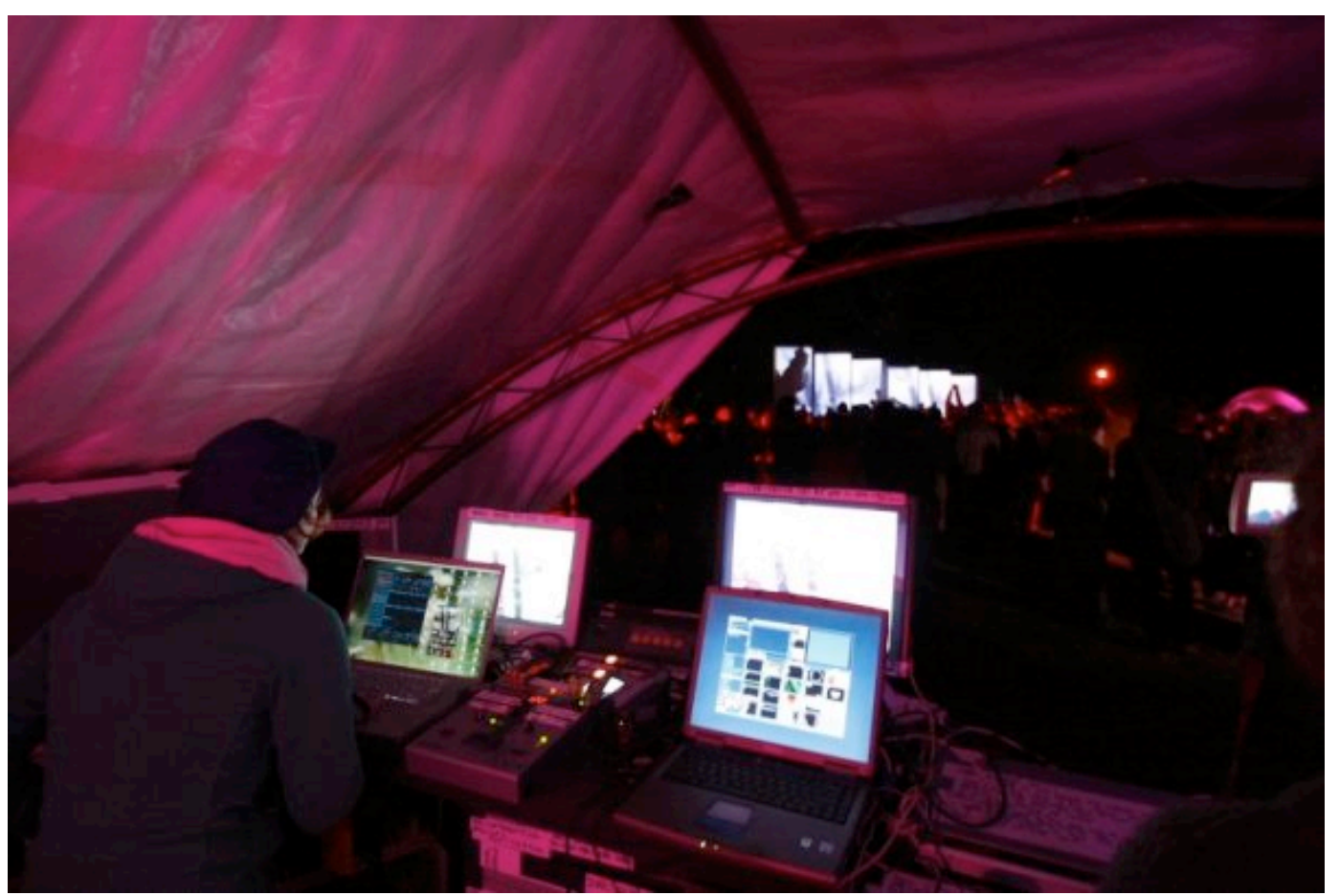

Figure 11: Author performing at outdoor festival. Photo: Dave Isdale. 


\section{CHAPTER 2}

\section{Case Study and Analysis}

This chapter will outline the process of designing and conducting an experiment that would enable investigation into recurring characteristics within a traditional VJ practice. This experiment concluded in four VJ performances, the results of which are contained in the accompanying DVD. Therefore this chapter outlines the methods of enquiry and design of this case study, which includes the rationale, the selection of participating $\mathrm{VJs}$, the compilation of a library of sample clips, and the establishment of rules and procedures for the experiment. Finally, this chapter will provide an analysis of this enquiry as well as its key findings.

\section{Purpose and Justification}

In this study I sought to investigate the process behind delivering a VJ performance. While working with and seeing other VJs mix, I have observed similarities in their practices. However, discussions regarding making samples are not as common as technical discussions on how samples can be mixed and altered in real-time, with software and hardware. I was curious to know if other VJs worked in a similar way to me, in terms of making samples for live mixing, and if there are any common denominators that influence the way in which VJs create and select visuals for live mixing. To conduct this study, I used my own practice as a catalyst for enquiry, in order to observe other veteran VJs' response to a given library of samples, to investigate the rules and conventions at play.

While there continue to be many diverse ways to present live moving image performances, many still exhibit some of the basic traditional principles of using loops, layering, montage, rhythm and selecting content from a library of clips. At the core these can be related back to Club type VJing. For these reasons, the project sought to emulate the most basic Club VJing elements: music, moving image samples (library of loops), software and projector screen and a VJ. It was important that all selected VJs had exhibited in their work an understating of the same traditional VJ principles from which my own practice had developed, in order to gain from the participants a reflexive response on these principles.

The first section of this chapter outlines the development of the case study, then synthesises and analyses the observations and interviews gathered throughout the process. What the development of the case study produced was a decision that there would be four VJs participating and they would be given a sample library of 108 samples to respond to in preparation for a performance to a 30-minute soundtrack. Using this controlled experiment I wanted to see if I could locate some key factors that impact on content creation for live VJ mixing based on traditional principles. 
Because the study would involve interaction with other performers as well as extensive interviews, this project needed clearance from the Victoria University of Wellington Human Ethics Committee. Approval was gained on $16^{\text {th }}$ September 2009, which made it possible to involve four VJs, interview them and record the results of a live performance (see Appendix 1). The process of submitting and subsequent approval from the Committee helped me frame my overriding question: What are some of the key factors that impact on the creation and selection of samples for traditional VJ mixing?

\section{Sample Library}

The following section outlines the rationale of producing the sample library from my own content, a description of what it contained and the results of a pilot test. As this investigation sought to identify some of the key factors that are associated with both creation and selection of content I therefore compiled a selection of pre-prepared samples. VJing is a practice that delivers content to a live public audience in real time. To continually deliver content over extended periods in these performances a VJ needs a large selection of samples to select from at any given time.

The samples I gave to the VJs came from my library for several reasons. Firstly, this avoided issues associated with copyright. Secondly, the samples in my own library were already tested clips. They ranged from samples made in 2001 to 2009, and all of them had been mixed during club, live cinema, or outdoor festival performance contexts. Thirdly, using my own samples would create a controlled experiment. Because each VJ would be accessing the same library, this would encourage the VJs to reflect on the content in this library, and encourage them to reflect upon how this may relate to his or her own content selections. This would assist me in isolating common factors that may arise as part of the participants' responses. Accordingly, this study was not directly about my work, but about other VJs' responses to it and, in the process, their explanations about their own practices.
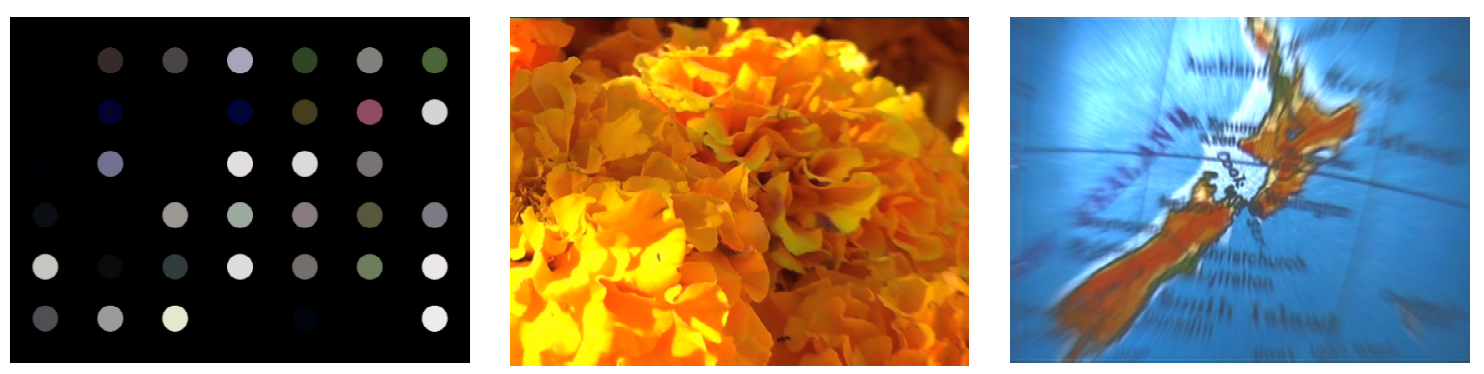

Figure 12 a, b \& c: Stills of moving image samples from case study library. Photo: Author.

The samples are of diverse origins and styles created over a period of the seven years. They include untreated, treated and animated clips that were all created using a series of different processes. The filmed footage was captured on either a Sony Video 8 Handy cam, Sony Digital 
Mini DV Handy Cam, or a Canon GL2 3-CCD Mini DV Camcorder, as these were cameras I have owned over this time. The editing software used was Adobe Premiere, and Final Cut Pro and After Effects. Any animated samples were created or manipulated with these same softwares, with some being further treated and recorded within the VJ software, Resolume Avenue. The filmed samples consist of full frame representational-untreated imagery such as landscapes, trees, coastlines, timelapse of sunsets, leaves, close ups of undulating water, droplets and tides. They also include a range of motion within the frame that has been achieved using hand-held camera movements and zooms. They include slow zoom into leaves, and flowers or shots from a moving vehicle, such as a car, a bicycle or even an aeroplane. Additionally, there are purely abstract images that contain coloured moving shapes on a black background. These were created within VJ software Resolume or by adding multiple effects and motion filters within video editing software. The larger portion of samples were processed in editing software to alter the colours and/or contrasts, or were completely transformed until there was no immediate relation to the original sample. This was achieved by adding a range of motion effects. Various samples, in particular those that contain figures, were edited and processed to appear as silhouettes. All the samples contain movement. This movement varies: from fast and chaotic to smooth and controlled, and this motion was often determined by the rhythms found within the frame when filming, using camera techniques or through the process of animating or manipulating the clip's timeline during postproduction. The duration of the samples varies from a few seconds to almost a minute.
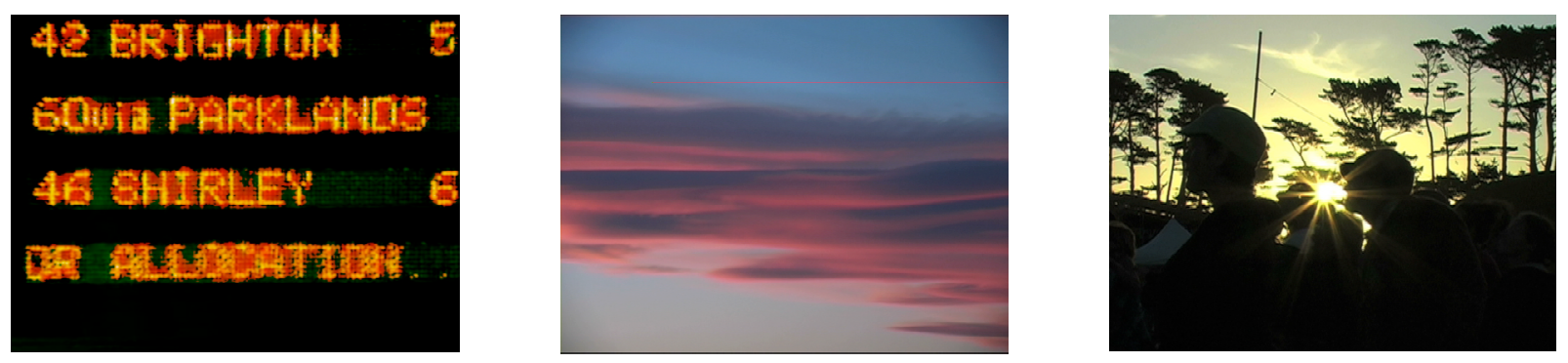

Figure 13, a, b, \& c: Stills of moving image samples from case study library. Photo: Author.

In a pilot test, I gave seventy-five of these samples on an external Mac hard drive to a VJ to use in an experimental live audiovisual event. At this event, the VJ mixed from the given library 'cold' (i.e. he had not viewed any of the samples prior to performing) for over one hour using the software Modul8 on a Mac laptop in response to improvised experimental electronic glitch music performed live to a gallery audience. This test revealed that after one hour of mixing the VJ had exhausted the library and had started to lose enthusiasm. Also the unfamiliarity of the samples added more uncertainty to the performance in a public setting. It was from this test that the additional fine-tuning of the study progressed. 

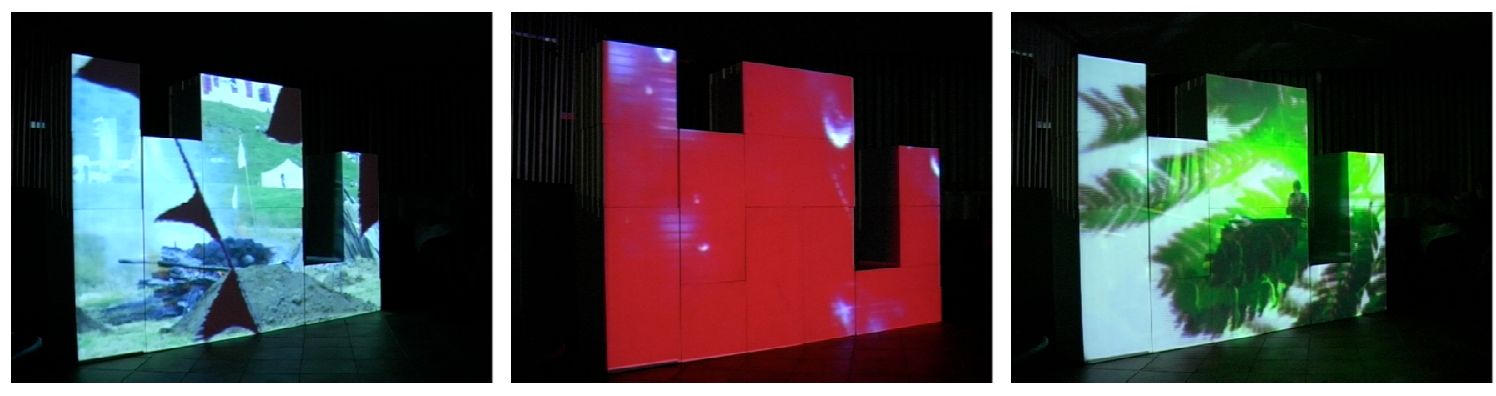

Figure 14 a, b, c: Inslallation view of Pilot test at Bundaburg Art Gallery 2009. Photo: Author

The duration of a VJ performance can range from sixty minutes to six hours, and it is rare that they are presented with the music that they will improvise to beforehand. However, because the purpose of this study focuses on the content, the pilot test revealed that a shorter performance time of twenty to thirty minutes, and set music, would suffice. It also became clear that more samples would be needed in the library in order to provide more options for the VJs to work with. Furthermore, this test revealed that it was important to give them an opportunity to become familiar with the samples before the performance.

\section{Compressing content}

The following section provides the rationale for the content compression choice that was made for this experiment. The main purpose of the pilot test was to assist in the process of constructing a sample library of content that could be accessed and easily translated into any hardware or software. Therefore it was not important to know specifically what hardware or software the VJs might be using. There is a wide range of hardware and software options for contemporary VJs to use for live performance. In this study it was important that the selected VJs use what they felt most comfortable performing with. Therefore, I chose to create the library in order to accommodate the possible various needs for each VJ. It was unknown to me at the time what software each VJ was intending to use for this study. So all the samples needed to be able to be opened in each VJ's computer, and the option made available for them to resize the samples if needed.

Live VJing is not a linear process; samples need to respond to being played forward, backwards, slowed down or sped up, sometimes at very rapid rates. Raw data files are often too large for effective real-time playback as they put too much pressure on the computer's ability to process these files while being manipulated and layered real time. Therefore, a raw data file needs to be compressed into a more manageable format. As most of the selected material for the sample library had already been formatted into VJ samples and ranged over multiple years, some data migration 
was needed so that the entire collection of samples was in the same format. For this, some samples needed to be recompressed. During isolated tests, I found that the Photo JPG codec at $680 \times 420$ frame size, 24 frames per second deinterlaced was the most suitable format, file size, ratio (4:3). Photo JPG can be opened on both a Mac and a PC, and when the file is rendered deinterlaced, all frames on the sample are rendered as complete and separate frames, which reduces the risk of artefacts appearing during rapid manipulation of the file. The frame size of $680 \times 420$ is small, but this size guaranteed a fast response to manipulation in real-time.

\section{Genre and duration for performance}

This section provides the rationale and process description for the accompanying soundtrack to which each VJ performed, and a justification for the genre and length of this soundtrack. I wanted to create a simulation of a VJ performance in reference to the traditional New Zealand VJ scene. However, during the pilot test and conversations with the participating VJs, I came to a realisation that, as this study focuses on the VJs' responses to the clips, executing this experiment during a public performance would add further complexities and technical complications that might not be relevant to the direction of this enquiry. Thus, I decided that each VJ would perform in isolation, so each VJ would not be influenced and possibly respond to the others' performances.

Figure 15: Dave Isdale's isolated performance. Photo: Tim Budgen

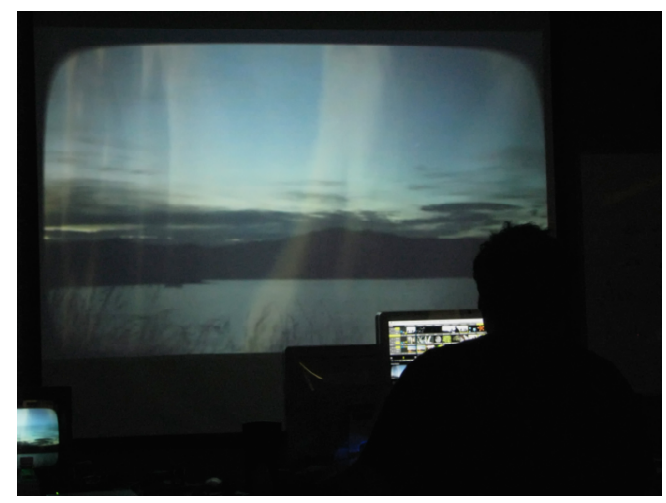

Similarly, the sound track would be pre-recorded. These two factors acted as 'controls' to the study. As previously mentioned, often VJs will play for minimum of one hour and up to six hours if needed. However, as this project progressed it became clear that a shorter time of around thirty minutes would be sufficient to enable the VJs to mix and get into a flow. Any shorter and there would have been less enjoyment for the artists, and a longer duration would have meant additional work for all parties involved that was not necessary in this instance.

A month was dedicated to seeking out and working on the audio track. One hundred music tracks were donated for consideration from over twelve artists through personal connections. All artists are based in New Zealand, and many have also had long standing connections with the Club and 
Outdoor Festival Scene. The initial collection ranged from hip hop, dub, drum and base, glitch, techno, ambient electronica, and jazz blues fusion. The final selection process happened during the compiling of the music on SoundCloud (Antipodean). Working online with a sonic arts student at Victoria University of Wellington, eight tracks were selected and mixed into a thirty-minute seamless piece. The motivation here was to try and simulate the continuous flow that a DJ might create, because traditional VJing is an improvisational performance in direct response to music. The final track has a slow build up, a climax and an ending that is drawn out. The intention here was to lead the VJ in exploring different tempos, styles and slowly wind down to a closing segment. The final tracks selected are all within the range of experimental electronica, glitch, dub and ambient electronica.

\section{VJ Selection Process and Rules}

In order to gather pertinent information about a diverse range of characteristics within VJ practice, I needed to invite veteran VJs to participate in this experiment. The following outlines the rationale and the rules for this experiment.

The VJ movement evolved from analogue to digital in New Zealand over the turn of the century. The content of this experiment's sample library is a direct result of my own practice, which developed based on traditional principles. It was important that these VJs had experience in traditional VJing, because that would mean they had the experience to know how to respond intuitively to my samples. Therefore, the VJs I sought to select needed to have exhibited the individual characteristics, experience, or skill sets listed below. Each VJ needed to have had extensive experience in a wide range of software, performance experience in a variety of genres and locations, and an understanding of hardware. Importantly they also needed to have had experience in sourcing and making their own content. They also had to display a work method that exhibited strong links to traditional VJ principles, such as using loops, layers, montage, motion, improvisation, rhythm, live mixing and a content library. Because the focus of this study is on content and what factors impact on the selection of the content, they needed to have experienced a number of diverse ways of presenting content in live environments, in other words, being aware of what works for them and what does not.

Through various performance situations I have worked alongside of, and seen the development of, the four VJs that were selected. This relationship has led to many conversations and supportive associations over the years. This was important because of the intimate nature of this project. The four selected VJs were Tim Budgen (Warp TV), Dave Isdale (Light Forms Media), Raylene Beals (rayzordoll) and Johann Nortje (VJ UJI). Tim Budgen, based in Christchurch, started exploring VJing in 1985. He has extensive experience in the club and outdoor festival scenes playing to diverse music types from house, drum and bass, trance, dub and while occasionally still plays in 
these areas he now specialises as a Live Band VJ. Dave Isdale, who also lives in Christchurch, has a background in photography and celluloid film. He works as a projection and installation artist with years spent performing at raves, warehouse parties, nightclubs, club nights and art galleries as well as outdoor festivals. Raylene Beals entered into VJing in 2003 from a background in experimental film and video, and uses her skills in dance clubs, outdoor festivals and experimental fringe performance arts projects often in collaboration with other performance artists. She currently resides in Wellington. Finally, Johann Norjte initially came to VJing exclusively from the drum and bass club scene in Wellington and in recent years expanded into VJing for bands, dub step, in clubs and is now specialising in interactive video mapping.

During the selection process I explained to them that they needed to participate in interviews and perform for a set duration using only content and music provided to them on a hard drive. Once I had received their confirmation to participate, a time line was arranged that would best fit their schedules, and allow for the travel time required to be able to conduct this test in both Christchurch and Wellington. I presented them with the following case study rules.

The VJs were allowed to use any software or hardware they wanted. They could alter the clips in the process of the mix anyway they wanted, could only use the clips in the library, and were not allowed to add any other content from outside the library or alter the clips in any way prior to the live mix other than to change size or codec. Each VJ would work in isolation from each other and the live mix would be done only once and recorded to tape. Each VJ would mix to the same sound track. The resulting output of the performance would be recorded to tape. I would be present at each performance, and record interviews with each VJ both before and after the experiment.

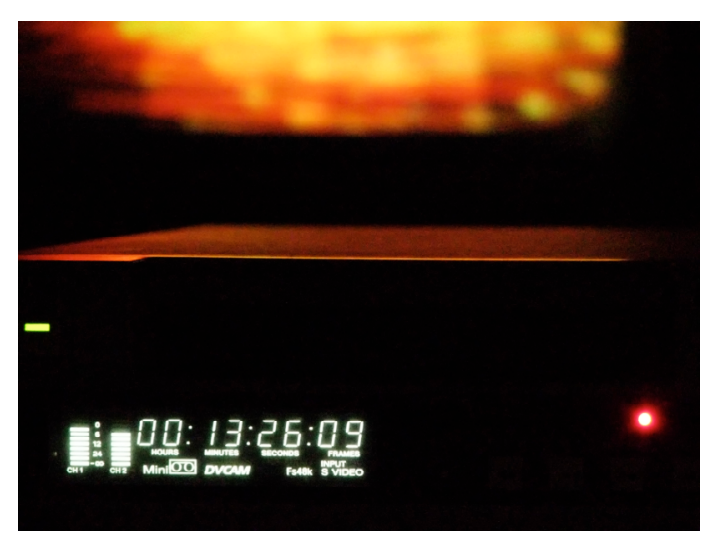

Figure 16: Recording performance. Photo: Tim Budgen. 


\section{Experiment and Analysis}

The following section provides a description of the experiment, outlines some of the key findings and how they were identified. It then provides a context for the accompanying DVD and offers the analysis of some key factors in relation to three samples used in the sample library, as well as discussion to the VJs' response to music.

In light of the issues arising from the pilot, the VJs received the hard drive two weeks prior to each scheduled individual performance. This would give them the option of becoming familiar with the format and the clips. The project's hard drive was partitioned into Mac and PC formats to ensure that each VJ could access the relevant drive compatible to his or her computer of choice. Each partition contained a duplicate copy of the folder containing 108 video samples and the 30-minute audio track.

By requiring the VJs to reflect on their own practice, within the constraints of responding to a given library of samples and music, this controlled experiment enabled me to isolate the similarities within their practices. The observations, interviews and casual conversations with the VJs discussing their processes in response to the given experiment started as soon as I made contact with the VJs after they received the hard drive.

After reviewing the semi-structured interviews, conducted both pre and post performance, a similar set of questions (see Appendix 2) was used in order to frame the discussions. At the end of this process, I was able to identify some of the key factors that impact on content creation and selection for traditional VJ mixing. These include: time, sight, memory, technology, availability, adaptability, efficiency, finance, environment, compatibility, loops, silence, form, rhythm movement, shape, colour and music.

The following sections outline these factors from the broadest to the most specific, then relates some of these factors in comparison to three of the samples from the library. Because this was an analysis of an entire process, from content creation to final performance, many of these factors are interwoven. The information conveyed in these sections is an accumulation of knowledge gained though in depth enquiries. 


\section{Sight, Memory and Time}

VJing is a visual performative practice. It is a type of communication that relies on sight, memory and time. This experiment highlighted these factors and the following is a description of how these were located.

When asked, "What did you do once you received the hard drive?" each VJ's response was similar. They opened each clip, and 'sighted' them. They opened the clips from the folder using an available codec carrier, such as Window Media Player or QuickTime media player. Each clip was sighted at least once, and some were 'played' with within the codec carrier. I witnessed this process with both Johann and Dave. Here the clips were played either once in their entirety, or they clicked on the timeline to make the clip jump around to see how it reacts. This led me to also ask the question: "How do you prefer to access you clips?" Again the responses were similar: the VJs stated that it would be preferable to be able to always see them first, and then add descriptive titles to each sample. Dave made a comment that highlights this: "At the end of the day I tend to look at what it is rather than the name of it".

An interesting factor that this study revealed was that after the VJs had looked though the clips once they did not return to the library again until at least the day or even a few hours before the performance. When questioned why this was, the most common response was that they did not feel they needed to. Once the clips were sighted, they felt they had a good understanding of the nature of the library. Through visual memory they would be able to recall these clips and spend time thinking about them prior to the performance. This type of mental processing using sight and memory turns out to be an important factor. When asked if they would have felt comfortable participating in the same project with either no time or less time to familiarise themselves with the content, each one concluded that, even though the library was not revisited, it was the time after sighting the clips and playing them over in their memory that contributed to a more enjoyable experience.

VJing as a practice is a time-based medium particularly in relation to the performance itself. However, this study has shown that the relationship to time also implies and necessitates seeing samples before the performance, rehearsing and playing the samples in the mind, so memory is used as a trigger during the performance. 


\section{Finance}

VJ practice generally relies on the performer owning most of his or her own equipment for content creation and playback. Yet this technology and software can be expensive, and with ever-changing performance environments these costs can be ongoing. As mentioned before, the samples I created were made using a number of devices to capture and edit and playback content. In discussions with each VJ, I asked them to describe some of the various technologies that they had used over the time that they had been VJing, and if any of the decisions regarding the costs of this technology affected their practice and if this in turn influenced how choices of creating content were informed. Each of them responded in a similar way. The general consensus seemed to be that they worked within their means and usually found something that provided them with what was both within their budget and satisfied their preferred way of working.

\section{Availability and Adaptability}

Adaptability is an important skill to have when VJing as there is no standardisation of technology in this practice. In this context, availability is used in terms of technology and how this contributes to the creation and selection of content. Adaptability refers to both the skills of a VJ to adapt to certain constraints, and to the development of content.

Each VJ had experience creating his or her own samples. When asked how this was done during the interviews, it became evident that each VJ adapted to whatever was available. The interviews revealed that while each had a preferred method of content creation (for example Dave liked to focus on film and stills while Tim preferred to use Flash and other animating software), each VJ would adapt to what was available. This is in regards to what they could afford to purchase, borrow or hire to assist in capturing and creating content, whether it be a high-end professional camera or a camera phone, scanned image, generative image created in open source software or the appropriation of imagery from film archives or popular culture. The interviews also revealed that all four VJs followed a similar practice of revisiting and reworking their existing clips to create new samples, as this material was always available for adaptation.

\section{Technology}

While Clubs are the most likely places to host traditional VJing, it is very rare that they will own any equipment to support the performance outside of providing a projector or a budget for the $\mathrm{VJ}$ to source equipment. Because of this there is little standardisation in a VJ rig and a VJ often has to source all his or her own equipment. This enables a VJ to choose to what they can afford and what feels right for them to use. For a good old fashion jam, Ray still on occasion chooses to use a VHS deck and tapes and a MX10. Johann is happy with just a laptop as long as he has his MIDI 
keyboard with him. Technology becomes a factor in the creation and selection of content for mixing in the following ways: from content creation there is a choice of image capture devices; for editing and playback a diverse range of hardware and softwares; and for mixing there is an interface that then connects with the projectors and screens. When asked how he was finding using the new version of Resolume on a Mac where he had previously used an older version on a PC, Dave's response was that "It's changing my workflow".

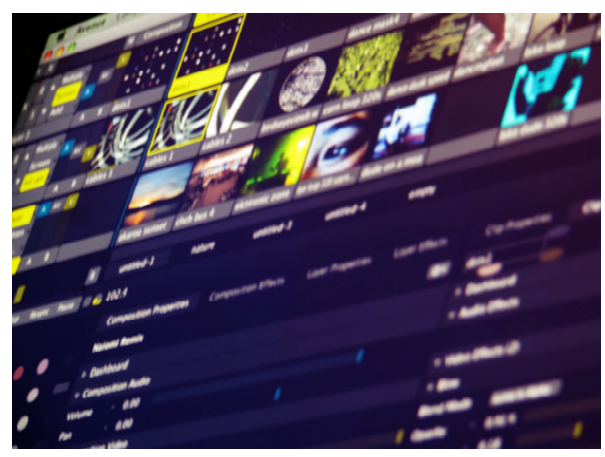

Figure 17: Resolume composition that Dave Isdale used during the case study performance.

This study revealed that, as with finance, the actual technology used does not alter the VJ's personal preference when considering his or her own aesthetic. Tim mentioned that, even if he was still using VHS today, the aesthetics of his content would still be the same but the process or workflow would be different. This statement was reiterated by Ray informing me that while she still like to incorporate VHS technology into her VJ sets from time to time the "process of creating content for VHS is very laborious and new technologies and softwares available today make the whole process much easier". Considering all these variables, availability, finance and ability to adapt are realities that most VJs are continually balancing. The key is to get everything working together into a flow, which is why the next factor in this analysis is compatibility.

\section{Compatibility}

This study revealed that content needs to be compatible with both the needs of the VJ such as the indented playback quality, and compatible with in any limitations of the technology they use. The software and hardware needs to be compatible with the resulting screens or projectors. At each point of these interactions the VJ needs to assess how the next item in the flow is going to be compatible with what is already present.

Compatibility is a factor when relating to codecs, as certain softwares and computers have preferred codecs and file sizes. This has an impact on content creation and selection because the content in its physical form, including aspect ratio and frame rate, as well as compression, needs to be compatible with the limitations of the software and hardware devices used during performance. After sighting the clips, both Ray and Tim found they had to recompress the samples from 
QuickTime to AVI as they both chose to perform in this experiment on older PCs. Ray expressed that "the QuickTimes don't play too good on my old PC". In addition these clips were then recompressed down to $320 \times 280$, a more manageable file size for the older computers and the older versions of softwares that they were intending to use. Ray said that she will always have to "bend to the limitations of the software" because throughout the whole process, if there is incompatibility at any point in the VJ's work flow, this impacts on the VJ's ability to have an efficient use of his or time while in performance mode. For example if the content files are too large for the computers to process in real time, the content will play at a slower rate and thus limit the VJ's ability to respond efficiently in time to the music.

\section{Efficiency}

Efficiency here refers to the allowance for maximum reaction time in locating, loading, mixing and layering and further manipulating loops in a live performance situation.

While memory and sight are contributing factors for locating clips on the fly, compatibility has a critical impact. While a QuickTime file can be opened on a PC, two VJs recompressed these clips to make them smaller with a more compatible codec. The motivation for this was to make the entire performance process more efficient.

If a clip that is hard to locate fails to play upon selection, is too large, or is not compatible with the system setup, it will, at worst, crash the system or simply be left out of the mix. Two VJs had computer crashes during the performance, and one VJ found that perhaps the codec was not the best for the software he or she was using. This meant that there was a lag in reaction time to the clips being selected. For the purposes of this study, as this was not a public performance, some of the VJs felt comfortable trying new things that they might not otherwise have attempted. This led to these resulting crashes, and thus further insight into how efficiency and compatibility are important factors. These technical errors are not evident in viewing the material on the DVD because, as previously mentioned, another skill of a VJ is the ability to adapt, and thus they continued to adapt to the given situation and still deliver a seamless performance.

\section{Environment (Context)}

All of the previous discussions help create an overview of the environment in which a VJ places himself or herself when considering creating and selecting content for live mixing. This section could also be called community or genre. Specifically limiting this analysis to club type VJing, environment in most cases relates to the genre of music. In outdoor parties the type of content used may be different to that of an indoor club. A VJ's set might be different for house, drum and base, 
electro, disco, hip-hop, ambient, etc. This change in context might mean that a VJ has to rework a prepared library of samples, or create new ones that might be more relevant to the music, and in turn, its audience. For example, Ray points out she would not play the same material for a both a "head banging psy-trance party and rock event". However she states that even within any changes in content, "at the end of the day, [the content] has to please me". This position was reiterated by Tim: it has to "sit right" with him and what he wants to present.

Of all the VJs, Dave Isdale was the only one that mentioned that my samples were close to what he himself would produce. The other three all found my work to be very different. What I concluded from this is that the environment that Dave and I have developed our practice is a different one to the other three VJs thus influencing our choice of content.

\section{Silence, Rhythm and Motion}

When I prepare samples, I often do so without reference to any music or sound. I have found this the best mode of working and have a specific reason for this. Sometimes people would question what type of music I listened to while making clips and were surprised when I said that I do not listen to any music when making content. During this study, I took the opportunity to ask if any of the other VJs did the same: they all concurred. Making a clip in silence means that there is more focus on the individual qualities such as the rhythm and tone of the clip. The rhythm and the movement can be seen as visual music. A club VJ is not usually aware of the exact music that he or she will be playing to, so fully exploring a clip's dynamics in silence opens up the VJ to a wider range of syncopative possibilities when later improvising with it. Each VJ interviewed for this study related that should a clip be made while listening to only one type of track, then that music informs that motion, rhythm, and tone of the clip. This potentially limits the clip to only being able to be played to that type of music. If a loop is made in silence then it is not associated with any type of music, which would foster improvisation later on. While working with a clip in silence, the VJs can assess the individual rhythmic qualities of that clip, or, as Tim puts it, see "visuals as rhythms".

The rhythm to which I am referring is within the clip itself. This does not refer to any rhythm that might be created while mixing though cutting, triggering or cross fading between clips during a performance or manipulating the speed, direction of the clip when performing. Rhythm in a clip is an important factor in a traditional VJ environment: just like a DJ, the VJ needs to keep the rhythm going. Nearly all the samples in my library have inherent movement. Rhythm and motion are often two of the main factors that inform the creation of a loop. Rhythm and motion can be captured during the process of filming, as illustrated by Dave's comment that he will "watch for the rhythm in the subject, for example, filming traffic flowing through and pausing flowing through and pausing". Though constructing clips in animation software or during pre-selection editing, the VJs 
can control the key frames at which motion is manipulated. The duration of the loop can create its own rhythm as well, and is also a contributing factor when considering where to place a loop into the mix.

\section{Form: Shape and Colour}

The formal qualities, such as colours and shapes, help with the placement of the clip in relation to layering. In particular, lightness and darkness, colours and tone. For example, Tim specifically considers these factors because this is how he layers his clips. As my clips were quite different to his, this meant he had to think about the process more in order to achieve a mix that still "sat right with him". When considering where a loop might go in a mix, each VJ mentioned that they all look for similar formal qualities, and as Tim mentions, in relation to "lights and darks". A silhouetted figure where the figure is featured as bold black and the rest of the image is white is an example that illustrates this point. The existing shapes within the clip also helped determine where it might be placed in a layer or in the flow of the mix. Most VJ software available today enables multi layering, where loops are played simultaneously over the top of each other. This follows on from traditional techniques such as using multiple playback devices through a hardware mixer. When discussing the use of layers in terms of how samples are placed in a composition using VJ software, most of the VJs considered the positioning in terms of top, middle, and bottom (or background). The four VJs have responded intuitively to the software and sample selections they have made over the years, but, with some discussion it was revealed that the formal qualities, such as shape or colour, help determine where the loop will appear in the layers. A silhouette, for example, would most commonly appear at the top and a full framed sample would be situated at the bottom, or as a background, and using cross fades or other mixing techniques within the software the VJ can move in and out, or up and down, through these layers.

Figure 18: Dave Isdale's composition during the case study performance showing silhouette of Sample A (figure20) positioned over the top of a full frame layer below Photo: Tim Budgen

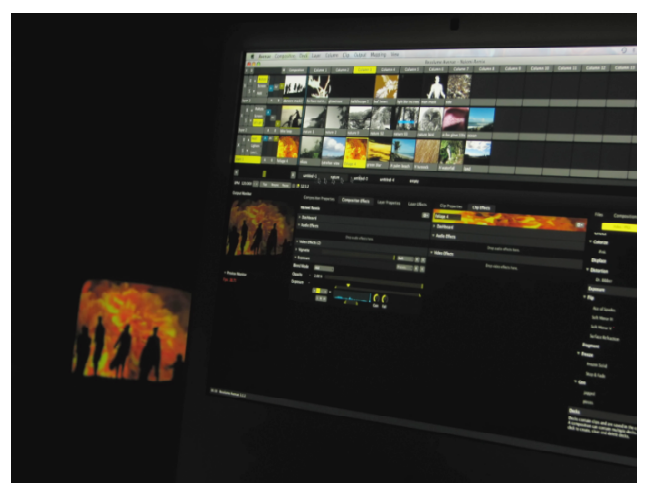

\section{Loops}

The traditional principles of loops, montage, and layers that previously were applied to a performance using a series of hardware devices can now all be contained within one of many available software options. In a performance, there is usually always a constant delivery of content on the screen, and the loop provides an image that is continuous. When using VHS, VJs would 
usually fill an entire 10 to 30 minute tape with content so that there would always be content running to go back to. In the move from VHS to digital files, the need to transfer this content to tape has ceased, and the content can be kept as short samples. All four VJs in this study mentioned that as the technology has changed a good proportion of their new samples have become shorter. Johann, for example, mentioned that over the time of developing his practice the majority of his samples have decreased in length from a few minutes to now only a few seconds. When creating a new sample, a key consideration is how it will respond to being looped. A seamless loop is one where the point at which the clip ends and repeats is not easily seen giving the illusion that is a constant and long playing sample. Another way to create the illusion of a loop is to set the playback option in the VJ software to bounce back, and therefore have the clip play forward and then backward over and over again. Thus a VJ thinks about these playback options when creating the sample. Dave explained that when creating "graphical imagery" in software such as Flash, After-Effects or 3D animation, it is easy to construct a sample where the beginning and end are the same so when they loop the content appears seamless. Johann and Tim both follow this method of content creation as a main feature of their practice. In contrast to this method of creating loops, Dave also explained that when filming for samples it is much harder to disguise the beginning and end points of footage. The shot may require editing to disguise the resulting meeting point. Because traditional VJing is in response to music, any apparent jump, cut or duration of clip can be used to the VJ's advantage to fit within the rhythms that he or she is responding to. Loops that are not seamless can be used to add a further syncopated rhythm. For example, this could bounce back and forward to match the beat or off beat of the music.

\section{The DVD in context}

The DVD featured at the end of three-part programme titled Dead or Live?

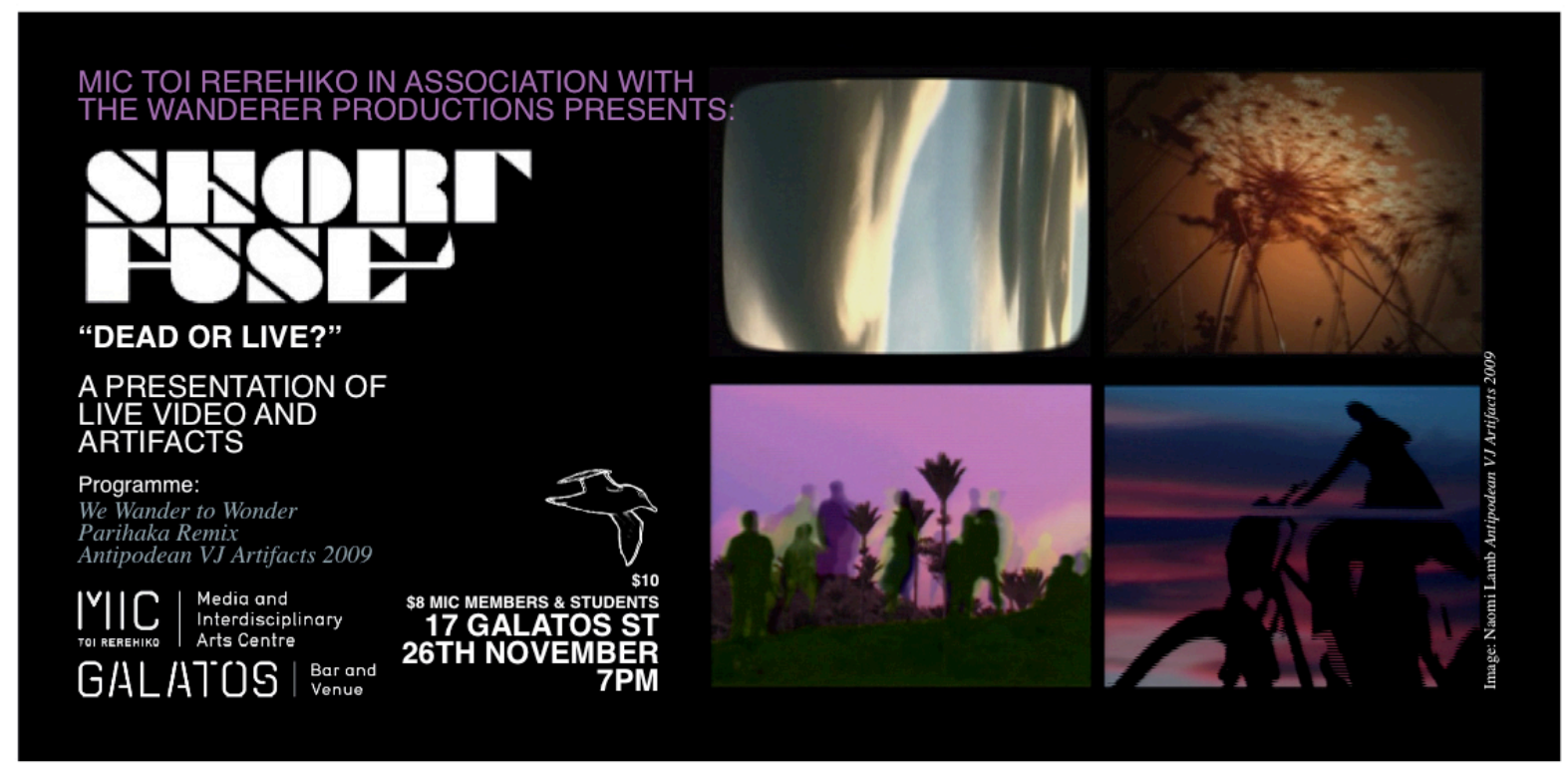

Figure 19: Flier for MIC Screening event that included Antipodean VJ Artifacts 2009 
The DVD contained at the back of this thesis has a specific format, the following discussion is a justification of how the material on the DVD came to be presented this way. The initial purpose of this case study was to analyse the results of the interviews conducted during this experiment. By taking four VJs into the environment of a controlled experiment, this created a means of comparison of how the four VJs used the samples in response to his or her own individual styles and methods. The DVD contains the recorded outputs of each VJ's mix. These are arranged in a grid so they may be viewed simultaneously to the same soundtrack. This arrangement was constructed for the purpose of a public screening held before the analysis I conducted. The DVD screened at the end of a public presentation that explored VJ contexts. The programme included a collaborative performance in non-linear narrative live cinema, and a solo audiovisual performance that explored the application of VJ methods into expanded documentary. This was held as part of a MIC Short Fuse programme in Auckland in 2009. MIC (Moving Image Centre) was a national audiovisual gallery that programmed events such as Short Fuse to showcase New Zealand artists.

For the context of this screening programme, the recordings of all four $\mathrm{VJ}$ performances were compiled onto the same DVD in a spatial arrangement. Because this DVD contained a presentation of four recorded live performances from New Zealand VJs, I gave it the title of "Antipodean VJ Artefacts 2009". As all four performances were conducted to follow the same sound track, it was feasible that they be arranged so that these documented outputs could be viewed simultaneously Previously the analysis relied on information gathered through interviews and partial observation of the performances in isolation. After this screening presentation, I was able to observe the similarities and differences in each VJ's response to this experiment and isolate how each VJ used the following samples. This enabled me to make the following observations.

In order to further highlight some of the factors that have been outlined, form, shape colour, rhythm, loops and layers, I will discuss how they relate to three selected clips from within the sample library. These samples can also be viewed in the performances contained on the DVD. These samples were chosen because they show characteristics that are important to the previous discussions. Through analysing these samples and their use by the four VJs, I will attempt to highlight some of the important considerations when creating content for VJing. In addition to this, I will end with a discussion on a short analysis of a section of the DVD as it appears in the spatial arrangement. This section will illustrate an overview of one factor that has yet to be discussed, that is music, and the VJs' responses to it. 


\section{Sample A: Form, Colour and Shape}
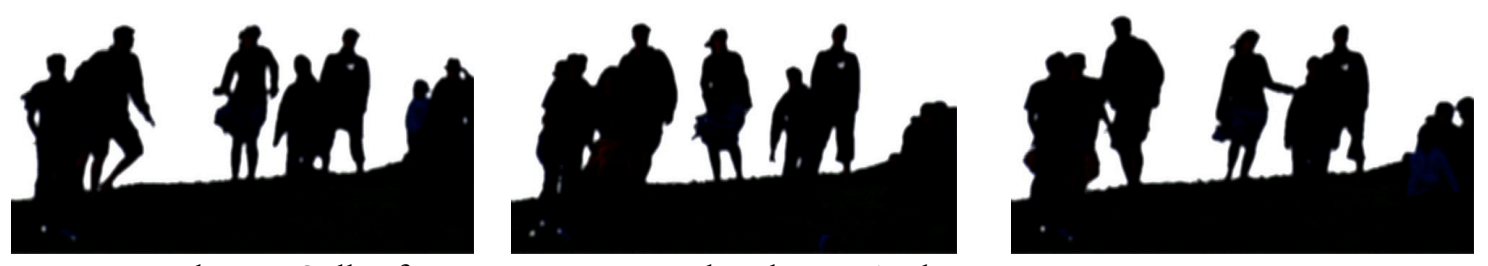

Figure 20 a, b \& c: Stills of moving image sample. Photos: Author

A characteristic that Sample A ((Figure $20 \mathrm{a}, \mathrm{b}, \mathrm{c}$,$) exhibits is what is referred to in this thesis as a$ silhouette, but is often commonly called a 'mask'. There are a number of masks that were contained in the sample library and used in similar ways in the performance presented in the DVD. Sample A, figures dancing on a hill, was used by all the VJs. Masks are most commonly bold areas of black and white or bold shapes of black or white with another dominant solid coloured form. The most dominant colour in a mask is often the one that is made transparent, to reveal the content from a layer that is placed underneath it. The 'mask' usually appears as a foreground layer, in this case, with the white layer removed. There are various techniques that can be applied to remove a dominant colour to reveal an underlying sample, such as Luma Key which is found in many compositing softwares, most VJ software and hardware mixers, and is commonly used by VJs who use traditional VJ techniques. In VJ hardware mixers and software "Keying is defining transparency by a particular color value (with a color key or chroma key) or brightness value (with a luminance key) in an image. When you key out a value, all pixels that have similar colors or luminance values become transparent" (Adobe Premiere). Because I follow traditional principles in my own practice, many samples were created accordingly. Each VJ had also used this same technique in his or her own practices and thus had used many of these masks in the way they were intended, that is to have one of the dominant colours removed to reveal a layer underneath. In this instance, the original clip was filmed on a three-chip camera on a tripod. The footage contains dancers on a hill with a solid blue sky behind them. The dancers thus create the dominant shape. I edited a sample taken from the original footage to create a beginning and ending of the loop and then I applied desaturation and contrast filters in Final Cut. When this sample was used each VJ removed the white, which left only the silhouette of the dancers viewable. This technique was repeatedly used. 


\section{Sample B: Rhythm, Movement and Seamless Loop}
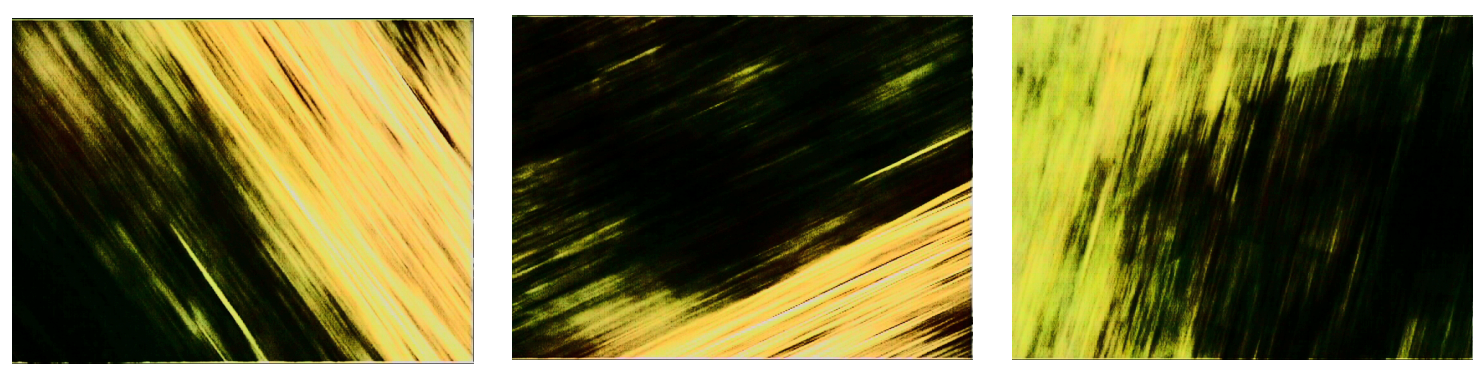

Figure 21 a, b, \&c: Stills of moving image sample. Photos: Author

Sample B (Figure $21 \mathrm{a}, \mathrm{b}, \mathrm{c}$ ) is an, abstract, rhythmic blur of green-yellow with high contrast lights and darks. This is also what Ray and Dave would refer to as "texture". While only used by three of the four VJs, it exhibited qualities that highlight the previously mentioned factors of rhythm, movement and seamless loop. Other abstract samples within the library were created using various different techniques, but this clip was filmed from a moving vehicle and a long sample of this footage was then treated in the editing software by applying a contrast filter which enhanced both the darks and lights. The purpose of a sample with these qualities is to add a continuous syncopated rhythm to any tempo or to keep the viewer engaged while the $\mathrm{VJ}$ is selecting other clips.

Three of the VJs mentioned that they like to use imagery such as water, clouds and fire, as these have natural organic motion and rhythms. This sample has an organic rhythmic quality and also rotates, adding further dynamic movement. Also, because of the high contrast, as with the masks, areas of this sample can be removed to reveal another layer underneath, as exhibited by two of the VJs' mixes. In this instance, even though there is no looping involved within the editing, this constitutes a 'seamless loop' because the organic rhythm, abstract form and length enable the clip to be repeated over and over again without any obvious breaks in the starting and ending of the clip to give the appearance that it is seamless. Even though Dave mentioned that using filmed footage sometimes creates difficulties in disguising the end and beginning points, this sample illustrates a comment he made that "if the content goes on for long enough there is a chance the audience won't be paying attention by the time it loops". Because of the formal qualities this clip contains such strong lights and darks and rhythm. This means the clip is suitable for use in any one of the layers. Each of the three VJs used this same sample in different layer provisions, so it appeared at different times either on top to later reveal a layer underneath, or in the middle, or behind a layer as a background. 


\section{Sample C: Background, Loop and Form}
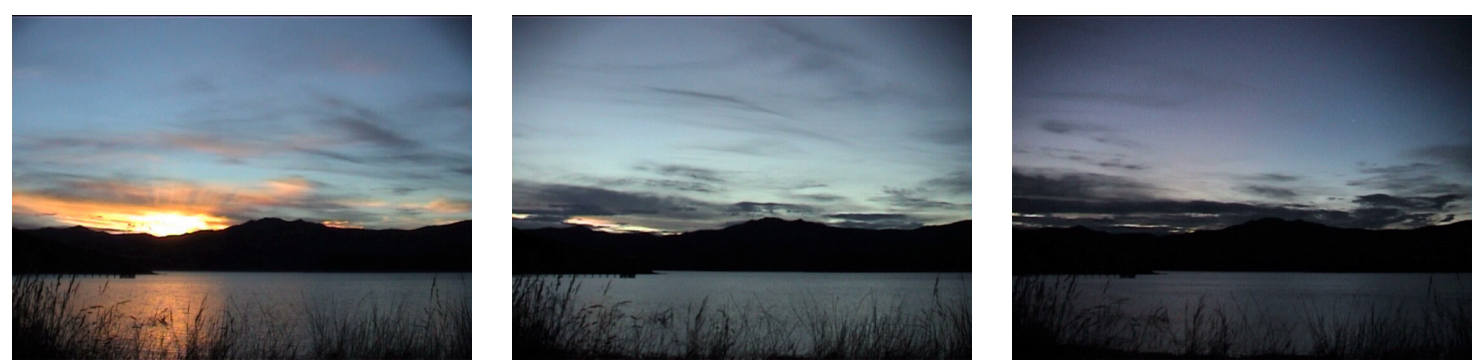

Figure 22 a,b \& c: Stills of moving image sample. Photos: Author

When considering making a loop the VJs will often think about where it may be used in a resulting mix. One of the factors in considering the form or shape and colour was where the loop would be placed in terms of top, as in a mask, middle or bottom. While a mask can give the VJ the option of choosing to make transparencies within the sample, when the formal qualities of colour and shape are not as clearly defined the loop can be considered "full framed". This means that when it is used the image contained within the clip extends to all four edges of the frame, which is why this is usually positioned as a background.

Sample C (Figure22 a,b,c), used by three of the VJs, represents a sample that would be most commonly used as a bottom or background layer. This sample is a time lapse of the sun setting past hills overlooking a harbour. Tim describes the importance of an efficient loop as "being a source that you can always come back to". Once a clip has been loaded into the playback interface or software it is important for the VJ to know that is will be running well and should there be a need to bring in or out multiple layers having a background layer means that there will always bee something presented on the screen even if for part of the performance this layer is obscured by the samples that a placed over top of it. A sample such as this makes a 'safe' option. It can be used on its own, or mixed over the top. It is not a seamless loop, so various techniques can be used to disguise the jump. Each VJ used samples such as this predominantly as intended, as a full frame image on its own or behind other samples. Dave mentioned that my content library exhibited similar aesthetic and form considerations, and he used this and a number of other full frame samples as backgrounds in his performance. In contrast, both Tim and Johann, even though both used this sample at either the beginning or end of their mix, stated that they would not normally have as many full frame samples in their libraries as they did not like square screens, and thus tried to pull the focus away from the edge of the screen, which motivated the predominance of black or negative space within many of their own samples. 


\section{Screen Grabs: Analysis of the Four}

\section{Mixes: Music}

Figure $23 \mathrm{a}, \mathrm{b}$ :

Screen grabs of the DVD at 00:20:36 and 00:23:38 along the timeline respectively.

Photos: Author
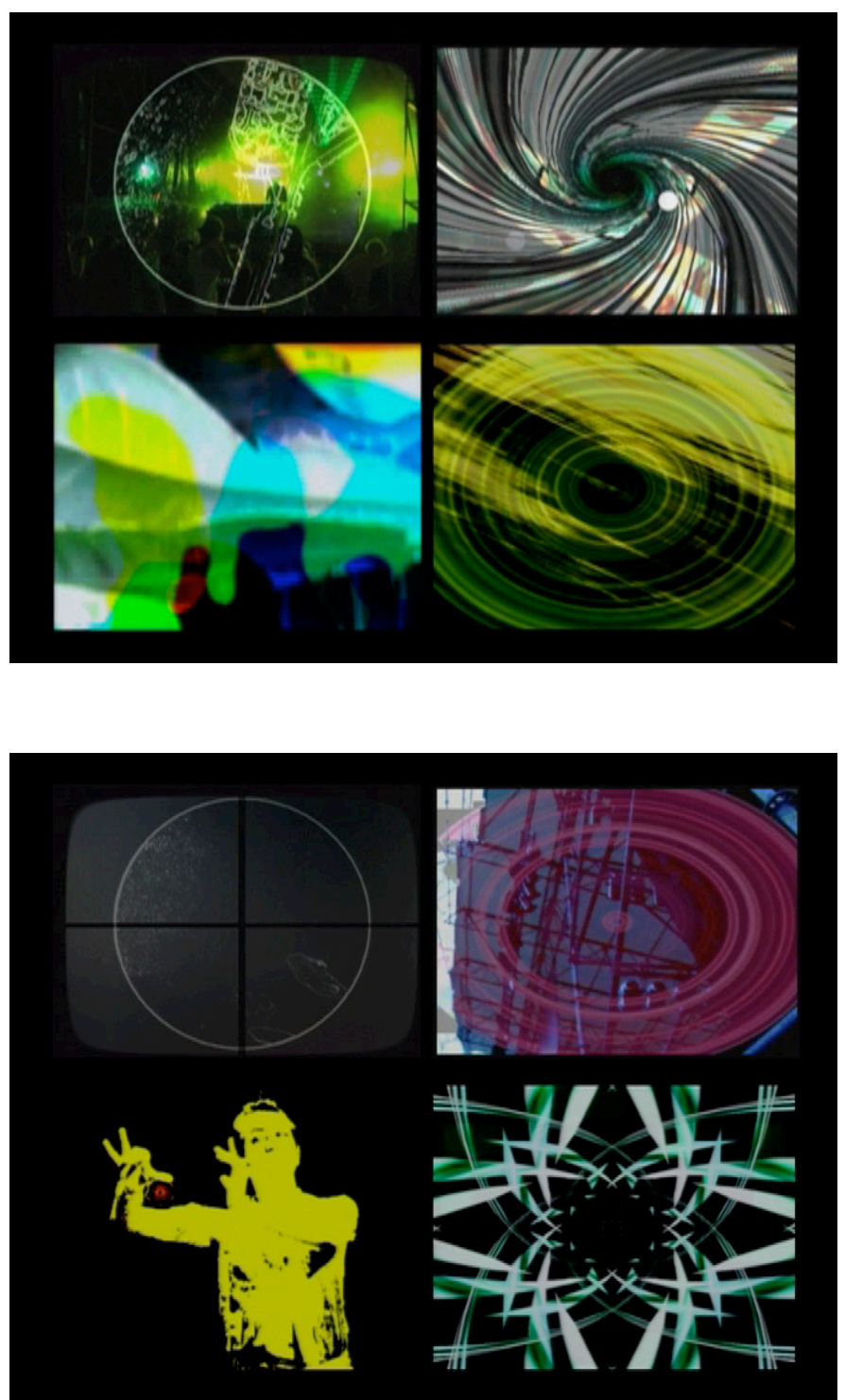

Through observation of all four videos, I was able to observe how each of the VJs responded to the music. Throughout the mixes, the VJs' choice of content and ways of mixing altered according to personal style and preference, but oftentimes they all responded in similar ways to the changes of tempo and tone of the music. The two screen grabs from the DVD (figure 23 a \& b) illustrate a point in the DVD where the tone and tempo of the music changes. The VJs responded accordingly. Each VJ used different samples, yet three ended up using similar circular forms and all started to explore a variety of effects within the VJ software. Three of the VJs responded quite similarly by using abstract, graphical samples, and the fourth VJ changed the colour effects, starting to use bolder colours, with blacks becoming more prominent in all four mixes. All the VJs added different effects at the same time that the music changed tempo. At this point, all four VJs started to exhibit a higher use of in-software effects, possibly in response to the faster, darker tone of the music. This observation highlights that, as a VJ moves though a performance in response to the music, the music does determine changes in both the content selection and the way the VJ mixes during the process. 
Music is a factor that influences a VJ's personal aesthetic. As previously mentioned, this study focuses on the genre of traditional VJing, which is most commonly found in clubs and outdoor dance parties. A VJ will often be drawn to or booked along with DJs to which his or her content would be suited. Thus the environment where the VJ works and the associated styles of music have a direct impact on his or her content choices. While the samples and music are not exactly what any of these VJs would choose, this case study shows how experienced VJs can successfully create an improvised performance given some time to sight and become familiar with the clips.

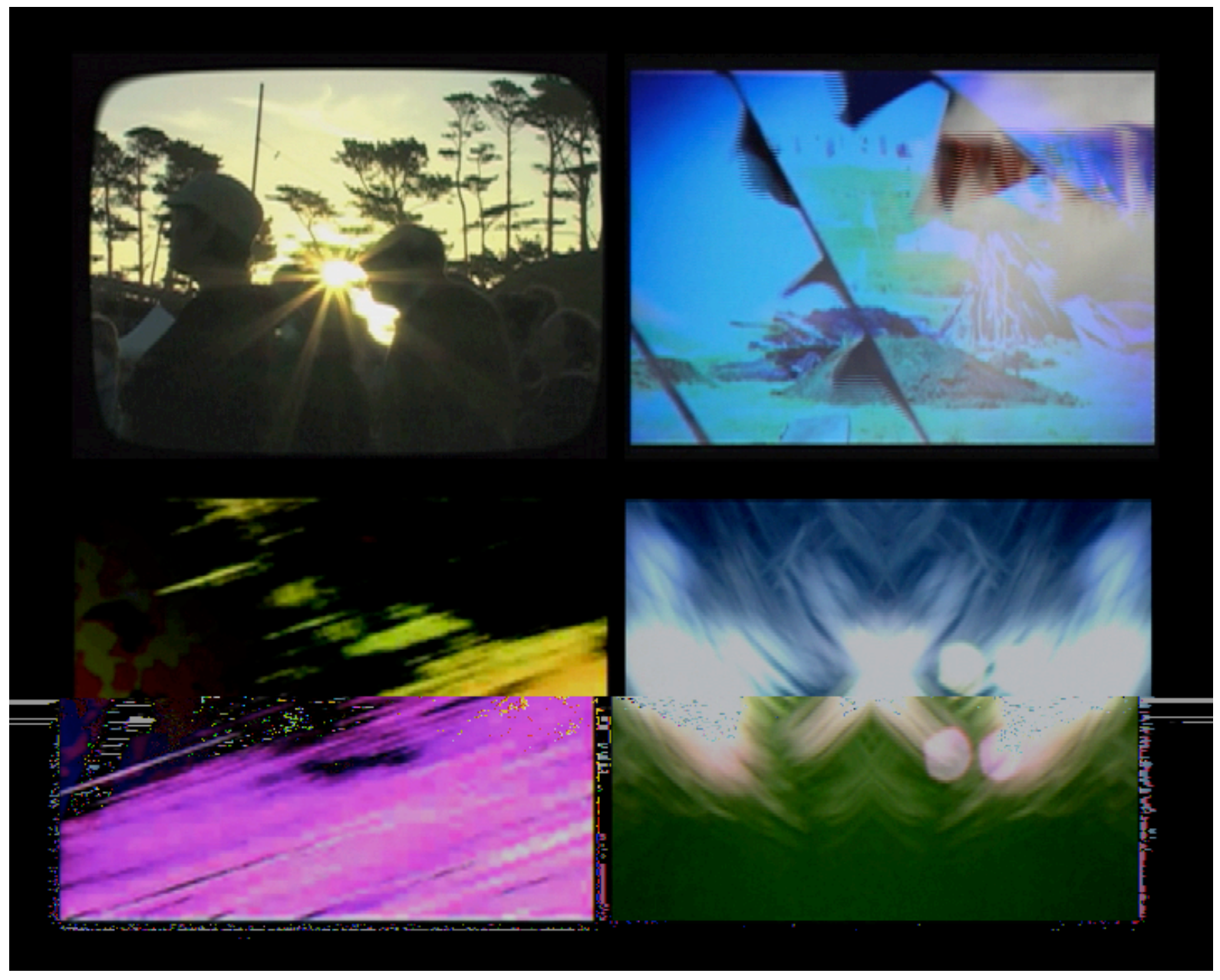

Figure 24: All four video records for the case study experiment. Photos: Author 


\section{Conclusion}

There are a variety of key factors that impact on content creation and selection for live VJ mixing. These include: time, sight, memory, technology, availability, adaptability, efficiency, finance, environment, compatibility, loops, silence, form, rhythm movement, shape colour and music.

What I have discovered in this study is that selection process actually happens before the performance while building a library (and consistently adding to it) prior to the mix. Building up the relationship with the library, in partnership with the context of the environment and the technology, informs the decisions during the performance. I have also found that some of the traditional principles of loops and layers are continuing factors that impact on the decisions of a VJ when considering creating a sample. Even though technology and finance were initially considered factors that impact on content creation and selection, it is evident that these mostly determine the changes in workflow rather than in aesthetic selection. Changes in hardware may have affected the way VJs work, but this experiment shows that VJs' personal aesthetic does not actually change over time. VJs adapt to the technology. The only constraint imposed on content may arise in terms of workflow and from making sure the codecs used are compatible, but, to quote Raylene, all VJs "bend to the limitations of software".

Factors such as environment, technology and finance affect workflow and process, while the formal qualities of the clip are influenced by the VJ's understanding of his or her own aesthetic and mixing style. Once a VJ knows what works best for him or her, he or she can set about creating and making selection processes before the material ever actually ends up in the mix. The main decisions occur during pre-production, while the $\mathrm{VJ}$ is working in silence, building each clip and developing its qualities to work within the mix. While the content in my sample library is quite different from the content the VJs might have chosen to source, select, sample, create and mix, the factors presented in this chapter are all things that each one of us considers for a performance.

Chapter One outlined some of the traditional principles and factors that are still in use in some contemporary VJ practices: as well as the characteristics within the Kiwi VJ scene. These traditional factors are layers, montage, motion, liveness, loops, improvisation, content for the screen, projections, remixing and rhythm. Chapter Two outlined an experiment that enabled me to locate additional factors that further contribute to the process and considerations of the selected VJs' development of a content library for VJ mixing. These additional factors include time, sight, memory, availability adaptability, efficiency, environment, compatibility, silence, and form: including shape and colour. 
This study showed that designing an experiment that acts as an initiative for focused inquiry was successful in provoking a dialogue between the researcher and the participants. The sample library provided a catalyst, which generated a disruption to the intuitive mode of working to which the selected VJs were accustomed. Tim Budgen explains this well when he says, "this project has been very good, I've enjoyed it, it has made me really think about my own work in ways I may not have before, I feel that I might go back and take another look at my own work". Thus, this process enabled me to discover further information that may not have otherwise been gathered and identified had I solely interviewed them about their personal practices. In this way, this case study has contributed to the understanding of VJing in New Zealand, positioning it within a wider global context.

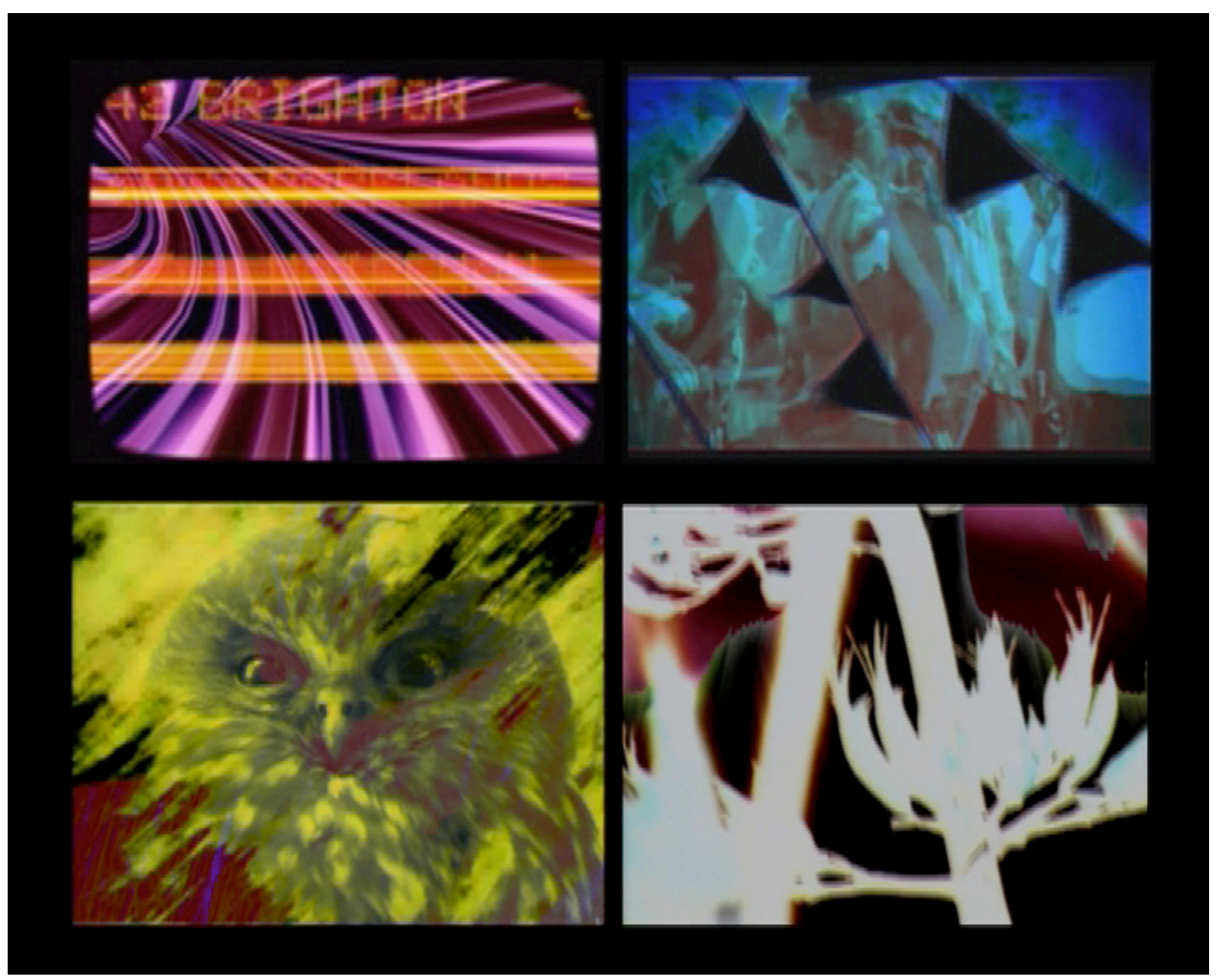

Figure 25: Screen grab from DVD: Photo: Author 


\section{References}

Adobe Premier (n.d) Retrieved from

http://livedocs.adobe.com/en US/PremierePro/3.0/help.html?content=WSAA75F4992DE3-4a4a-BCD1-09D6AC9D703D.html

Alpine Unity. (2009). Retrieved from http://en.wikipedia.org/wiki/Alpine Unity

Antipodean. (2009) Retrieved from http://soundcloud.com/vjvideogirl/antipodean-audio-track

Black, K. \& Cook, J. (2003). Vjzoo. Retrieved from http://vjzoo.com/tewhatisvj.htm

Christchurch 90s Rave Scene. ( n.d.). Retrieved from http://www.facebook.com/group.php?gid=202050693219\&ref=search

Clifford, A. (2008). Interdisciplinary moments: A history in glimpses. In S. Brennan \& S. Ballard (Eds.) The Aotearoa digital arts reader (pp. 114-135). Auckland, NZ: Aotearoa Digital Arts and Clouds.

Cooke, G. (2010). Startmaking Sense: Live audio-visual media performance. International Journal of Performance Arts and Digital Media, 6 (2), 193-208.

Creative Commons. (n.d.). Retrieved from http://www.creativecommons.org.nz/

Dance on a Rock. (n.d.). Retrieved from http://www.danceonarock.com/about.html

Faulkner, M. (Ed.). (2006). VJ, audio-visual art + vj culture. London, UK: Laurence King Publishing Ltd.

Flipbook. (n.d.) Retrieved from http://www.flipbook.info/history.php 
Hodgson, M. (2006). VJing in New Zealand: a personal perspective. In M. Faulkner (Ed.). VJ, audio-visual art + vj culture (pp. 148-149). London, UK: Laurence King Publishing Ltd.

Horrocks, R. (2009). Art that moves: The work of Len Lye. Auckland, NZ: Auckland University Press.

Intersect. (2009). Retrieved from http://test-tube.tv/intersect/

Jaeger, T. (2005). Live cinema unraveled. Handbook for live visual performance. San Diego, CA: University of California San Diego.

Kiwi VJs. (n.d.). Retrieved from http://www.facebook.com/group.php?gid=95448985089\&ref=search

Manovich, L. (2001). The language of New Media. Cambridge, MA: MIT Press

MIC (Moving Image Centre). (n.d.) Retrieved November 2009 from http://www.mic.org.nz/events/screen/shortfuse-november-09/)

Mikela, M. (2006). Live cinema, Language and elements. (Unpublished MA thesis in New Media, Helsinki University. Helsinki, Finland).

Nortje, J. (2010) Persistance of Illusion, Using spatial illusion as a visual performance mechanism. (Unpublished MDes Thesis in Media Design, Victoria University, Wellington, New Zealand).

Outdoor Styles. (n.d). Retrieved from http://www.outdoorstyles.co.nz/

Project shown*. (n.d.) Retrieved from http://www.thewandererproductions.co.nz/?q=node/18 
Rush, M. (2005). New media in art. (New edition). London, UK: Thames and Hudson.

Scott, G. (Producer). (2006). Aspiring [Television documentary]. Wellington, NZ: Gibson Group.

Sonics from Scratch. (n. d.) Retrieved from http://www.sonicsfromscratch.co.nz/

Spinrad, P. (2005). The VJ book. Los Angeles, CA: Feral House.

Splore. (2010). Retrieved from http://www.splore.net/

The Gathering. (n.d.) Retrieved from http://www.thegathering.co.nz/

User manual_(n.d.)._Retrieved from http://www.usersmanualguide.com/panasonic/digital av mixer/wj-mx50

Virkhaus, V. (2006). Concert visuals. In M. Faulkner (Ed.). VJ, audio-visual art + vj culture (pp. 36-41). London, UK: Laurence King Publishing Ltd.

Vision (2001). Retrieved from http://obscure.co.nz/events/vision

VJ Theory (n.d.) Retrieved from http://www.vjtheory.net/ 


\section{Appendix 1}

Ethics approval
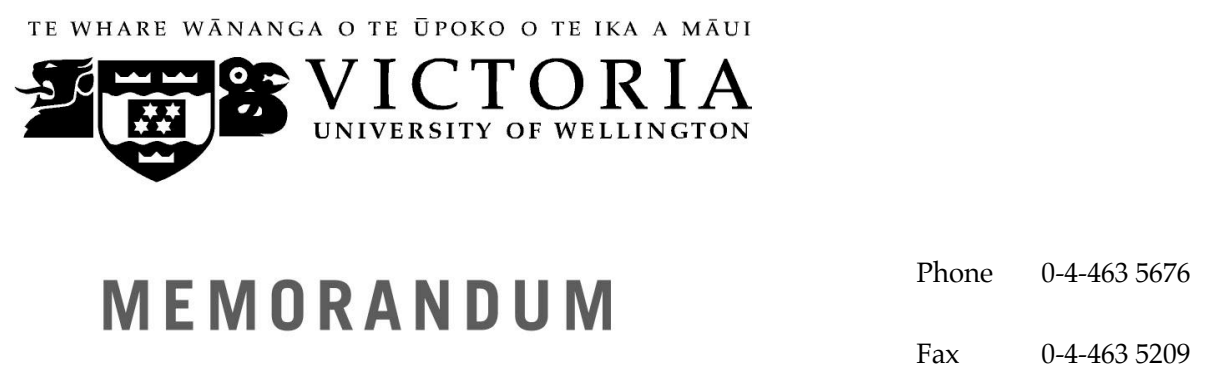

\begin{tabular}{l|l}
\hline TO & Naomi Lamb \\
\hline COPY TO & Morgan Barnard \\
\hline FROM & Dr Allison Kirkman, Convener, Human Ethics Committee \\
\hline
\end{tabular}

\begin{tabular}{l|l}
\hline DATE & September 16,2009 \\
\hline PAGES & 1 \\
\hline
\end{tabular}

\begin{tabular}{l|l}
\hline SUBJECT & $\begin{array}{l}\text { Ethics Approval: No } 16917 \text { - VJ Remixing (a New Zealand case } \\
\text { Study) }\end{array}$ \\
\hline
\end{tabular}

Thank you for your application for ethical approval, which has now been considered by the Standing Committee of the Human Ethics Committee.

Your application has been approved from the above date and this approval continues until 1 October 2010. If your data collection is not completed by this date you should apply to the Human Ethics Committee for an extension to this approval

Best wishes with the research.

Allison Kirkman

Convener 


\section{Appendix 2}

\section{Pre-prepared questions that helped direct the discussions and interviews with the VJs}

- In what contexts do you VJ?

- How do you define a good video sample?

- Was there enough content to hold your interest?

- How long do you need to work on the content before you feel comfortable improvising with it?

- How does this library differ from your own?

- What elements make a good library for VJing?

- How do you prefer to access your clips?

- With what and where do you source your content?

- What are the content considerations you think of when creating or selecting clips?

- (ie subject matter, treatment, tone, movement, loops, quality)

- What was challenging with this project?

- What would make this a better experience for you?

- How does your software affect the mix and the treatment of the clips?

- How does the hardware affect the mix and the treatment of clips?

- What are the main factors that contribute to your own creation of content?, ie context, software, etc.

- Over the course of your VJing history has the technology changes influenced the way you $\operatorname{mix} / \mathrm{remix}$ ? 